\title{
Machine Learning Approaches for Accurate Prediction of Relative Humidity based on Temperature and Wet- Bulb Depression
}

\author{
Qi Luo ${ }^{1, *}$, Manouchehr Shokri², Adrienn Dineva ${ }^{2, *}$ \\ ${ }^{1}$ School of continuing education, Chengdu Normal University, Chengdu, Sichuan Province, 611130, China; \\ ${ }^{2}$ Institute of Structural Mechanics, Bauhaus University Weimar, D-99423 Weimar, Germany; \\ manouchehr.shokri@uni-weimar.de \\ *Corresponding authors email: a.dineva@ieee.org
}

\begin{abstract}
The main parameters for calculation of relative humidity are the wet-bulb depression and dry bulb temperature. In this work, easy-to-used predictive tools based on statistical learning concepts, i.e., the Adaptive Network-Based Fuzzy Inference System (ANFIS) and Least Square Support Vector Machine (LSSVM) are developed for calculating relative humidity in terms of wet bulb depression and dry bulb temperature. To evaluate the aforementioned models, some statistical analyses have been done between the actual and estimated data points. Results obtained from the present models showed their capabilities to calculate relative humidity for divers values of dry bulb temperatures and also wet-bulb depression. The obtained values of MSE and MRE were 0.132 and 0.931, 0.193 and 1.291 for the LSSVM and ANFIS approaches respectively. These developed tools are user-friend and can be of massive value for scientists especially, who dealing with air conditioning and wet cooling towers systems to have a noble check of the relative humidity in terms of wet bulb depression and dry bulb temperatures.
\end{abstract}

Keywords: wet-bulb depression; relative humidity; ANFIS; artificial neural network; LSSVM

\section{Introduction}

The main factors to control the quantity of moist air are the temperature and pressure. As the air temperature increases, the amount of water vapor is also increasing [1,2]. The widely applied parameter in practice for determining a characteristic of air is the dry-bulb temperature, which is known as the air temperature. Since the obtained air temperature by a thermometer is not function of the air humidity, it is named as dry bulb [3,4]. Conversely, the wet-bulb temperature obtained by a wet thermometer which is affected by the airflow. The rate of evaporative cooling that is a type of cooling with the capability of removing the moisture from a surface usually is measured by a thermometer. The main distinction of the wet bulb and dry bulb temperatures is their amount. Except at relative humidity equal to 1, all the time the quantity of the dry bulb temperature is more than temperature of wet bulb $[5,6]$. According to guidelines, the difference between the wet bulb and dry bulb temperatures is known as the wet-bulb depression [3,5]. The wet-bulb depression is an important term to determine the relative humidity and this will be shown at following. The quantity of water vapor in the air in comparison with the full saturation is known as relative humidity. In other words, relative humidity can be defined as the ratio of the amount of water vapor in the air at 
the known temperature to the maximum value that the air can hold at this temperature [7]. The relationship between wet bulb depression and relative humidity is an important key in the numerous practical applications for engineers who dealing with air conditioning and wet cooling towers systems. In more details, as indicated in the equation (1), the relative humidity means that the water vapor partial pressure in the air-water mixture relative to the equilibrium water vapor pressure at the similar temperature [8].

$\gamma=\frac{P_{w}}{P_{w s T_{a}}}$

The approximation of wet bulb temperature is performed through the equation presents below:

$P_{w}=P_{S T_{w b}}-B^{\prime}\left(T_{a}-T_{w b}\right)$

In addition, the pressure difference between the wet-bulb and dew-point conditions usually is estimated by the following equation.

$P_{s T_{w b}}-P_{w}=\Delta\left(T_{w b}-T_{d p}\right)$

Equation (4) obtains from the combination of equations (2) and (3):

$$
\begin{aligned}
& B^{\prime}\left(T_{a}-T_{w b}\right)=\Delta\left(T_{w b}-T_{d p}\right) \\
& T_{w b}=\frac{B^{\prime \prime} T_{a}+\Delta T_{d p}}{B^{\prime+\Delta}}
\end{aligned}
$$

Here, $B^{\prime}$ is identified as indicated in equation (6) and it denotes the psychometric constant for the air.

$$
B^{\prime}=\frac{1006.925\left(P-P_{S} T_{w b}\right)\left(1+0.15557 \frac{P_{W}}{P}\right)}{0.62194 h_{f g}}
$$

Moreover, the term of $h_{f g}$ which is known as the latent heat can be determined from the following equation.

$$
h_{f g}=1000\left[3161.36-2.406\left(T_{w b}+273.15\right)\right]
$$

According to the proposed correlations, the wet-bulb depression is estimated by:

$$
W_{b d}=\frac{0.1533 T_{a}+5.1}{0.9}(1-\gamma) W_{b d}=\frac{0.1533 T_{a}+5.1}{0.9}(1-\gamma) \quad\left(\text { for } \mathrm{T}_{\mathrm{a}}, 0-30{ }^{\circ} \mathrm{C}\right)
$$

and

$$
\left.W_{b d}=\frac{0.4355 T_{a}+5.1}{0.9}(1-\gamma) \quad \text { (for } \mathrm{T}_{\mathrm{a}}, 0-110^{\circ} \mathrm{C}\right)
$$

Where the term of $T_{w b 1}$ is introduced as:

$T_{w b 1}=T_{a}-W_{b d}$ 
In addition, when $T_{w b 1}<T_{d p}, T_{w b 1}$ is identified as:

$$
T_{w b 1}=T_{d p}+0.5
$$

Accordingly, the term $\Delta$ can be approximated by the equation (3) through substituting $T_{w b 1}$ for $T_{w b}$, which is known as:

$$
\Delta=\frac{P_{S} T_{w b 1}-P_{w}}{T_{w b 1}-T_{d p}}
$$

These equations should be solved through iterative procedures.

Briefly, to determine $\mathrm{T}_{\mathrm{wb}}$, the amounts of $B^{\prime}$ and $\Delta$ are substituted into equation (5). Then, these procedures are continued by substituting $\mathrm{T}_{\mathrm{wb} 1}$ for $\mathrm{T}_{\mathrm{wb}}$ with modified $B^{\prime}$ and $\Delta$ quantities. Consequently, the new approximations are replaced in equation (5) and these procedures continue so long as $\mathrm{T}_{\mathrm{wb}}$ converges with good accuracy.

Bahadori et al. proposed an Arrhenius-type asymptotic exponential function model for predicting the relative humidity in terms of dry bulb temperature and wet bulb depression. Their correlation is easy-to-apply and has good agreement with real data [9]. This correlation is formulated in below and its coefficients can be found in its reference.

$$
\begin{aligned}
& \ln \left(H_{R}\right)=a+b D_{w}+c D_{w}^{2}+d D_{w}^{3} \\
& a=A_{1}+\frac{B_{1}}{T}+\frac{C_{1}}{T^{2}}+\frac{D_{1}}{T^{3}} \\
& b=A_{2}+\frac{B_{2}}{T}+\frac{C_{2}}{T^{2}}+\frac{D_{2}}{T^{3}} \\
& c=A_{3}+\frac{B_{3}}{T}+\frac{C_{3}}{T^{2}}+\frac{D_{3}}{T^{3}} \\
& d=A_{4}+\frac{B_{4}}{T}+\frac{C_{4}}{T^{2}}+\frac{D_{4}}{T^{3}}
\end{aligned}
$$

Beside this correlation type method, application of statistical learning approaches can be a benefit in the present case. There are several types used embranchments of statistical learning approaches, i.e. fuzzy logic, ANN, SVM, and ANFIS [10-14].

In the present study, the potential of adaptive ANFIS, LSSVM and two kinds of artificial neural network which are known as the MLP and RBF structures was investigated to calculate the relative humidity based on wet bulb depression and dry bulb temperature. Then, a databank of data points was gathered from the reference to achieve this end (see Table 1) [15].

\section{Theory}


ANFIS is a kind of the neural network method that has value for applying in function approximation problems $[16,17]$. In other words, an ANFIS structure is a combination of the knowledge obtained from the artificial neural network and fuzzy logic system. Each ANFIS structures contain some parameters called membership function parameters (MF) that should be optimized using optimization algorithms. Thus, owning to this special structure of the ANFIS, it is more systematic and its dependency on actual data is less than other machine learning approaches such as the ANN [18]. The illustration of typical the ANFIS can be seen in Fig. 1.

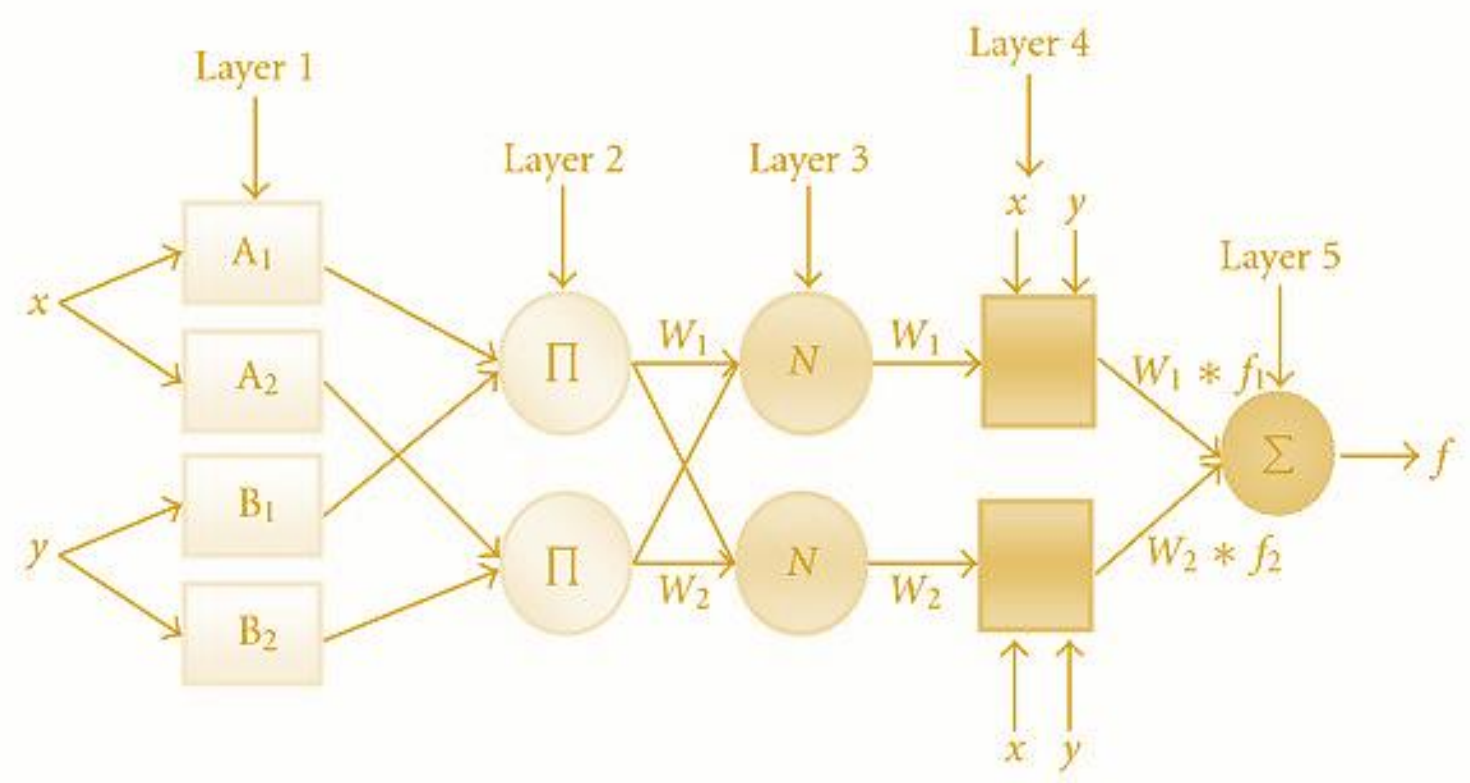

Fig. 1 Scheme of the typical ANFIS.

A common ANFIS form is created basically from the five layers. In this layer, some nodes present and they are specified by their node functions. The relationship between each layer basically can be performed by internal connections. As demonstrated in this figure, the layer's inputs are supplied by the preceding layer's outputs. It should be noted that the Sugeno type is used as fuzzy system of the ANFIS method.

In more details, if the inputs contain two parameters and their notifications are $\mathrm{x}$ and $\mathrm{y}$, and output consists of only one parameter, namely $\mathrm{f}_{\mathrm{i}}$, the ANFIS rules can be introduced as [16]:

- First rule: If $x$ is $M 1$ and $y$ is $N 1$ then $z$ is $f 1(x, y)$

- Second rule: If $x$ is $M 2$ and $y$ is $N 2$ then $z$ is $f 2(x, y)$

Here, the terms $\mathrm{M}$ and $\mathrm{N}$ stand for the fuzzy sets and the first order fuzzy inference system outputs are shown by $f_{i}(x, y)$.

The adaptive nodes present in the first layer are defined as:

$O_{1, i}-\mu_{M_{i}}(x)$ for $i=1,2$ 
$O_{1, i}-\mu_{M_{i-2}}(x)$ for $i=3,4$

where $\mu(y)$ and $\mu(x)$ represent membership functions.

In the next layer, each node is constant and indicates with notification $\pi$.

$o_{2, i}=\omega_{i}=\mu_{M_{i}}(x) \cdot \mu_{N_{i}}(y)$ fori $=1,2$

where $\omega_{i}$, refers to the rule's firing strength.

The third layer contains nodes that are constant and indicate with notification $\mathrm{N}$. The firing strength is normalized by their node functions. To that end, the firing strength value of $i^{\text {th }}$ node is divided to the all firing strength summation.

$O_{3, i}=\overline{\omega_{l}}=\frac{\omega_{i}}{\sum \omega_{i}}=\frac{\omega_{i}}{\omega_{1}+\omega_{2}} \quad$ for $i=1,2$

The fourth layer has nodes that are adaptive and indicate with square shapes.

$O_{4, i}=\bar{\omega}_{i} \cdot f_{i}$ for $i=1,2$

$f_{1}$ and $f_{2}$ stand for the fuzzy if-then rules. The formulations of their rules are represented as follows:

- Rule 1: if $x$ is $M 1$ and $y$ is $N 1$ then $f_{1}=p_{1} x+q_{1} y+r_{1}$

- Rule 2: if $x$ is $M 2$ and $y$ is $N 2$ then $f_{2}=p_{2} x+q_{2} y+r_{2}$

Where, $\mathrm{p}_{\mathrm{i}}, \mathrm{q}_{\mathrm{i}}$ and $\mathrm{r}_{\mathrm{i}}$ refer to the consequential terms.

In the last layer, overall output is calculated through:

$O_{5, i}=f_{\text {out }}=\sum_{i} \bar{\omega}_{i} \cdot f_{i} \quad$ Overall output

However, the output is characterized as a linear combining the consequential terms. The last output is specified by:

$$
\begin{array}{r}
c f_{\text {out }}=\varpi_{1} f_{1}+\varpi_{2} f_{2}=\frac{\omega_{1}}{\omega_{1}+\omega_{2}} f_{1}+\frac{\omega_{1}}{\omega_{1}+\omega_{2}} f_{2}= \\
\left(\varpi_{1} x\right) p_{1}+\left(\varpi_{1} y\right) q_{1}+\left(\varpi_{1}\right) r_{1}+\left(\varpi_{2} x\right) p_{2}+\left(\varpi_{2} y\right) q_{2}+\left(\varpi_{2}\right) r_{2}
\end{array}
$$

A typical ANFIS is learned by the hybrid algorithm, which is the combination of the least squares approach and gradient technique. Consequential terms can be determined by the least squares approach in the forward pass and the signal of errors are propagated in the backward pass [19]. In this study, we used the capability of a genetic algorithm to calculate parameters of the ANFIS method.

\subsection{Least Square Support Vector Machine (LSSVM)}

The supervised LSSVM approach was the first created by Suykens and Vandewalle in 1999 devoted to the function approximation and regression problems. If the inputs represent by $\mathrm{X}_{\mathrm{i}}$ ( here in, wet bulb depression $\left(\mathrm{D}_{\mathrm{wb}}\right)$ and temperature $(\mathrm{T})$ ) and the output indicates with $\mathrm{Y}_{\mathrm{i}}$ (relative humidity $(\mathrm{RH})$ ), the typical LSSVM nonlinear function can be defined as formulated at the following [20]: 
$f(x)=w^{T} \phi(x)+b$

Here, the term $\mathrm{f}$ refers to the target $(\mathrm{RH})$ and inputs ( $\mathrm{D}_{\mathrm{wb}}$ and $\left.\mathrm{T}\right)$ connections, $\mathrm{w}$ applies for the vector of m-dimensional weight. Furthermore, $b$ is the bias of the term. Owning to the theory of minimization, the regression problem usually is solved by considering following expression:

$\min J(w, e)=\frac{1}{2} w^{T} w+\gamma \frac{1}{2} \sum_{k=1}^{N} e_{k}^{2}$

In addition, following limitations should be regarded:

$y_{k}=w^{T} \varphi\left(x_{k}\right)+b+e_{k},(k=1,2,3 \ldots N)$

Here, $c$ refers to the margin parameter and $e_{k}$ represents the error variable of $x_{k}$. According to the straightforward derivations of the LSSVM by Suykens, the following expression will be obtained $[20,21]:$

$f(x)=\sum_{k=1}^{N} \alpha_{k} K\left(x, x_{k}\right)+b$

Owning to the good performance of the radial basis function or RBF, it is usually employed as kernel function in regression faults. This kind of function can be indicated by the following equation:

$K\left(x, x_{k}\right)=\exp \left(\left\|x-x_{k}\right\|_{2}^{2} \sigma^{-2}\right)$

In Eq. (24), the term $\sigma^{2}$ refers to the squared bandwidth and it should be determined through optimization. To this end, the mean square error (MSE) of the real value and LSSVM output is assumed as the cost function of the optimization method. This cost function is formulated as following as:

$M S E=\frac{\sum_{i=1}^{n}\left(P_{\text {rep } / \text { pred }_{i}}-P_{\text {exp }_{i}}\right)}{n s}$ 


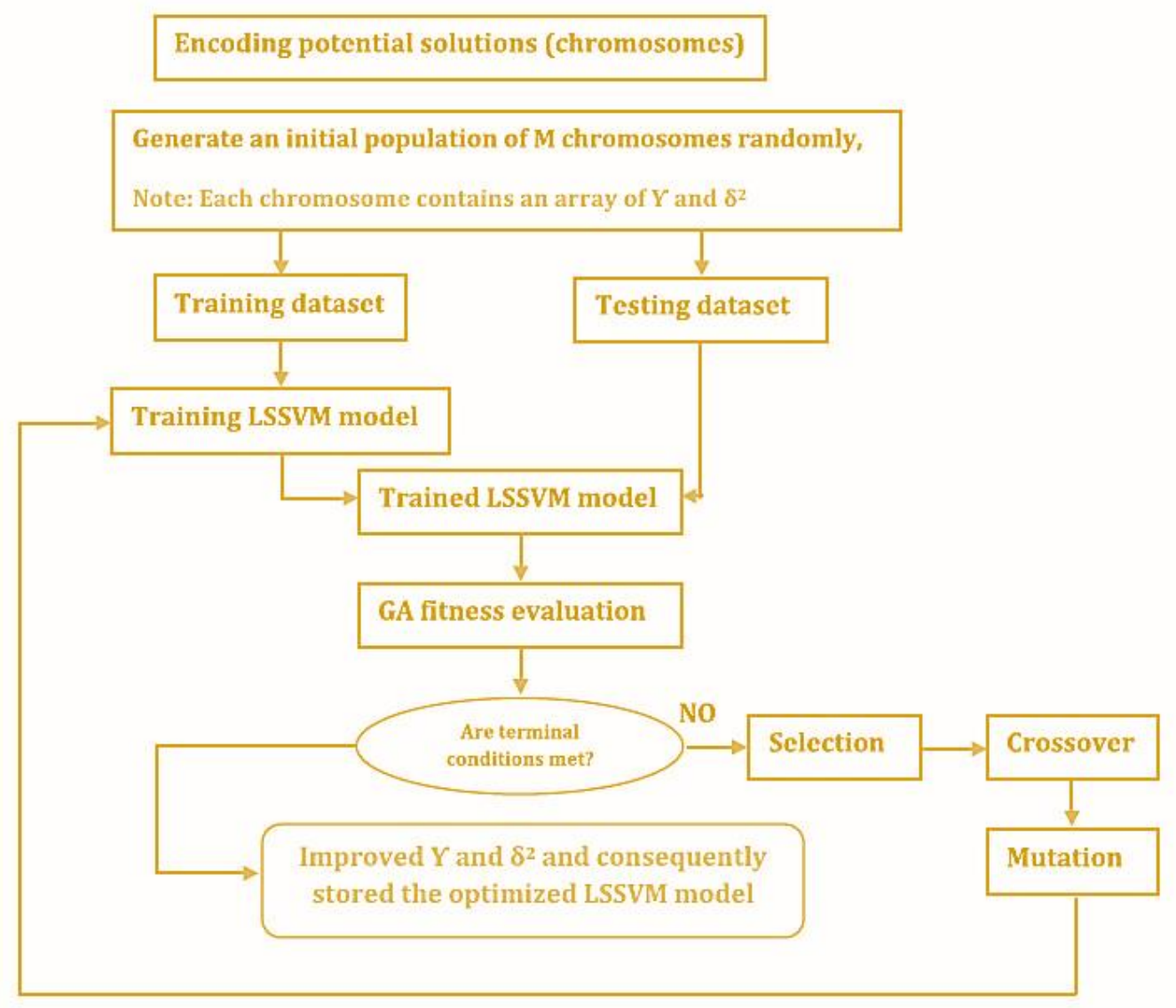

Fig. 2 The typical configuration of the LSSVM approach.

In which $\mathrm{P}$ is the $\mathrm{RH}$, subscripts rep/pred and exp denote the estimated and measured $\mathrm{RH}$, correspondingly, and ns stands for the number of samples. The goal of this approach is to determine optimal dependent variables (i.e., $\sigma^{2}$ and $\gamma$ ). In this regard, several optimization methods including Back Propagation (BP), Particle Swarm Optimization (PSO), Genetic Algorithm (GA), Hybrid Particle Swarm Optimization and Genetic Algorithm (HPSOGA), Imperialist Competitive Algorithm (ICA), and Unified Particle Swarm Optimization (UPSO) can be applied. In the present work, we used Genetic Algorithm to optimize $\sigma^{2}$ and $\gamma$. The flow diagram of this approach is shown in Fig. 2.

\subsection{Artificial neural network (ANN)}

As observed in Fig. 3, the multilayer perceptron (MLP) neural network is created from input, hidden, and output layers. In this structure, the number of hidden layers may be one or more. Each layer consists of some neurons that their number should be specified by optimization techniques or trial and error procedures. In order to learn the ANN model, the parameters are adjusted till minimum square error (MSE) is obtained. In MLP structure of ANN usually, is optimized by back-propagation technique. The optimized number of iterations which is named by epochs must be used to determine the weights and biases to overcome undertraining and overtraining problems. These problems lead to obtaining poor results at testing phase $[22,23]$. 


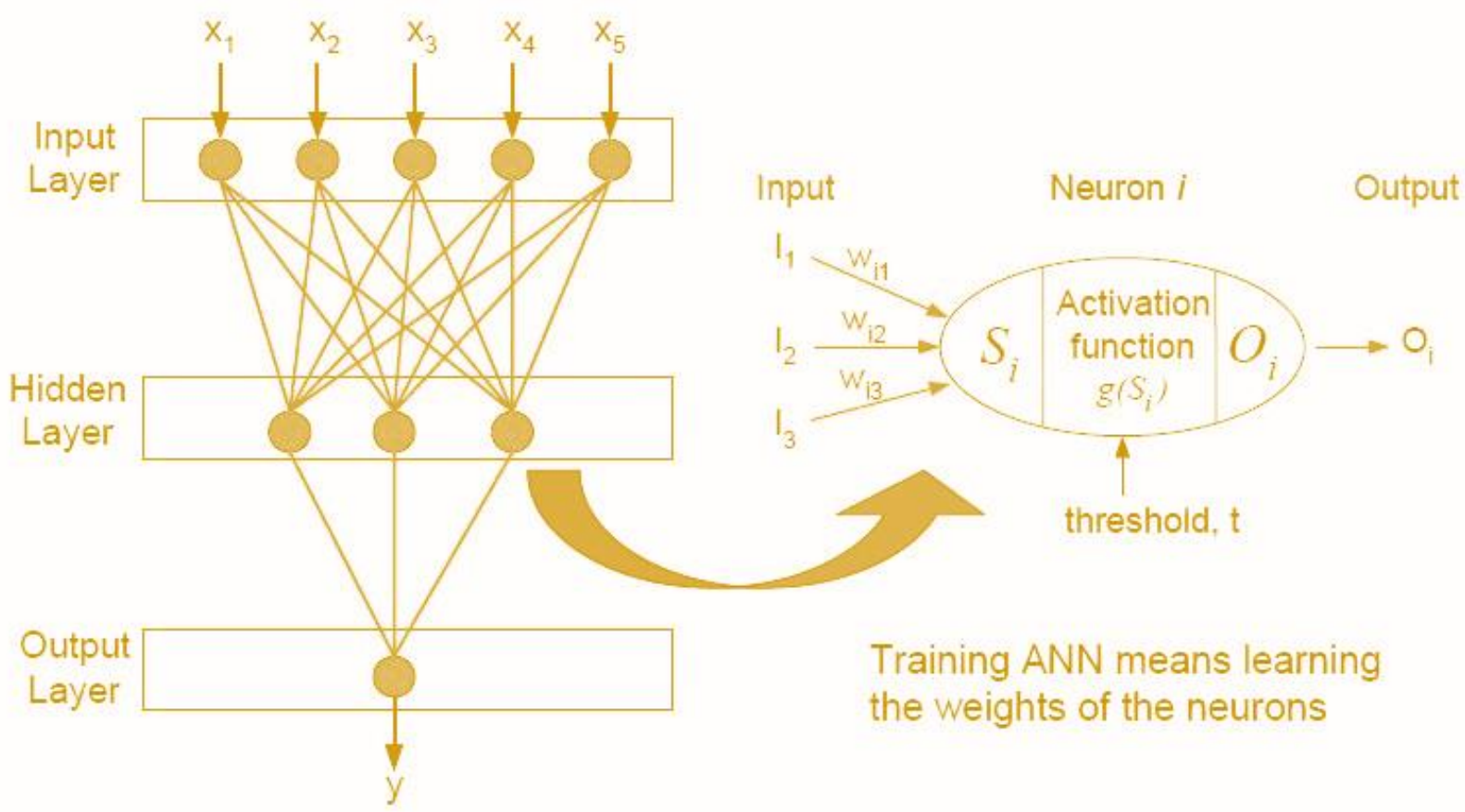

Fig. 3 Structure of typical MLP artificial neural network.

There is a distinct computation in the structure of the MLP and RBF, while their utilizations are similar. Owning to the simple design of the RBF frameworks (see Fig. 4), they have more advantages than MLP structure. This distinction makes the RBF structure more capable for generalization of outcomes [24].

RBF framework is a kind of iterative function estimator which is used the concentrated basis functions. In order to create a model, this framework uses a supervisory training technique that is a kind of feed-forward neural network.

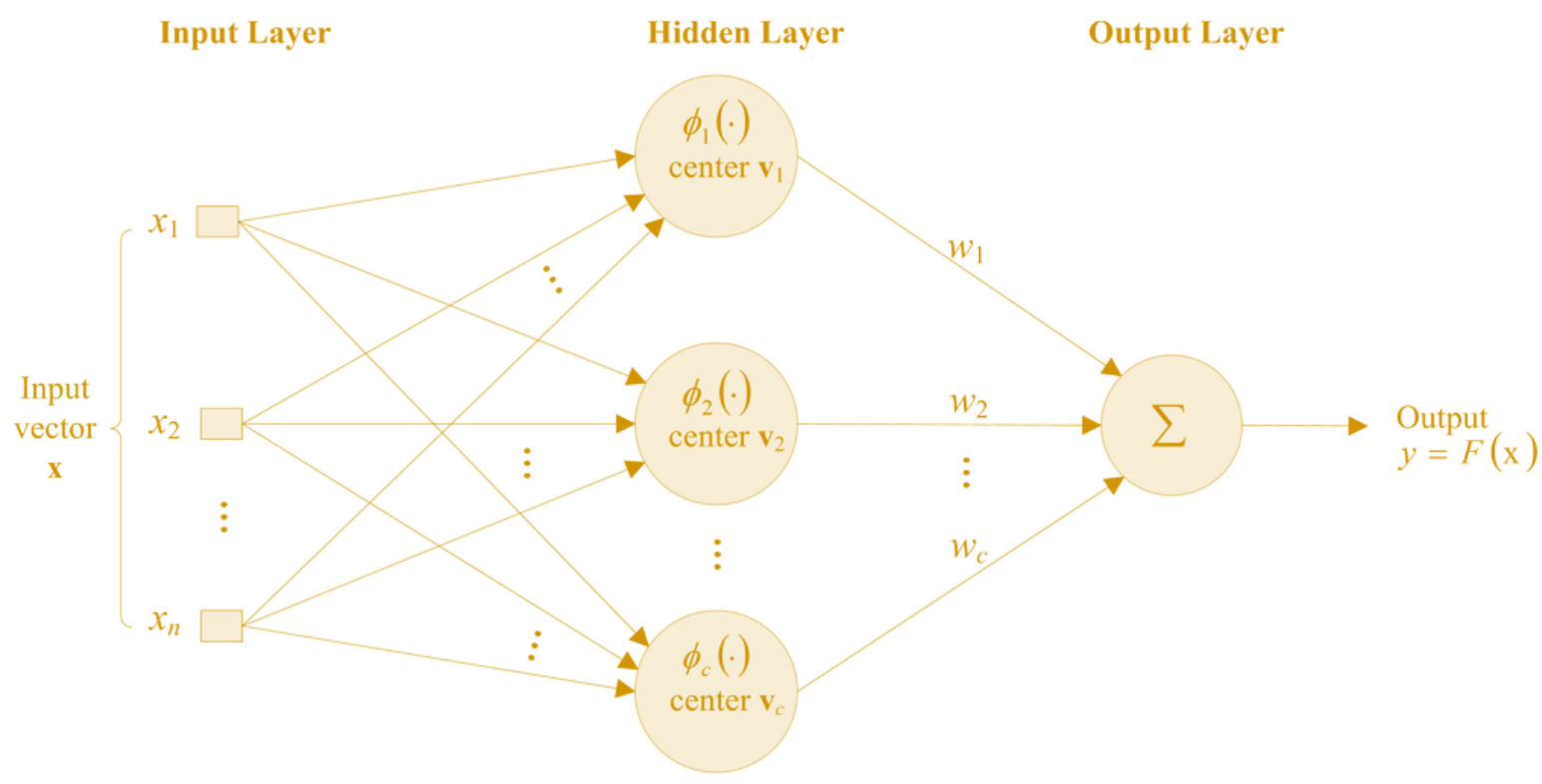

Fig. 4 Scheme of typical RBF-ANN. 
Thanks to RBF structure, its computations is much simpler and quicker than MLP structure. The RBF configuration is similar to the conventional regularization framework. Three advantageous features of regularization framework are expressed as follows:

- Its capability to estimate any multivariate nonstop function on a compressed domain in order to a reach satisfactory outcomes through utilization of an adequate number of components.

- The best solution is determined with minimization of a function that indicates its oscillation.

- Owning to the linear configuration of unidentified coefficients, there is the best estimation feature.

As shown in above two figures, the scheme of the RBF is nearly similar to the MLP. It has three layers; an output layer, an input layer, and a hidden layer. However, the main distinctions of these two configurations including:

- RBF framework uses the easier construction than MLP.

- There is much simpler training process in RBF framework than MLP.

- MLP structure acts globally and its outcomes are specified through the relationship between all the neurons, while the RBF structure acts locally and its outcomes are determined by identified hidden units in particular local accessible areas.

- The classification manners in these frameworks are different. Separation of each cluster is performed by the hypersurfaces in the MLP structure, while this task is done by the hyperspheres in RBF configuration.

\section{Development of Models}

The precise experimental relative humidity values are required to evolve aforementioned models. In this study, we used the relative humidity, wet bulb depression, and temperature that have been recorded in the reference and their ranges were presented in Table 1 [15].

The next stage is the selection of inputs and target variables of models. To that end, the wet-bulb depression and temperature are given as inputs and relative humidity is regarded as the target parameter. The database used in this study consists of 330 data points. This data set should be divided into two datasets namely, training and testing sets, to train and test the capability of proposed models.

Table 1 Ranges of data employed in the present study.

\begin{tabular}{|c|c|c|}
\hline Wet-bulb depression, C ${ }^{0}$ & Dry bulb temperature, Co & Humidity \\
\hline-10 & $1-30$ & $7-92$ \\
\hline-5 & $1-30$ & $8-93$ \\
\hline 0 & $1-30$ & $8-93$ \\
\hline 5 & $1-30$ & $9-93$ \\
\hline 10 & $1-30$ & $10-94$ \\
\hline 15 & $1-30$ & $11-94$ \\
\hline
\end{tabular}




\begin{tabular}{lcc}
\hline 20 & $1-30$ & $12-94$ \\
25 & $1-30$ & $13-94$ \\
30 & $1-30$ & $14-94$ \\
35 & $1-30$ & $16-95$ \\
40 & $1-30$ & $17-95$ \\
\hline
\end{tabular}

The training data set contains 248 data points which are approximately $75 \%$ of total data points and the remained data points were used for testing. In addition, these data points were normalized within the ranges of -1 and +1 to achieve better performance with these models.

As mentioned, in the current work, four models based on machine learning concepts including, the ANFIS, LSSVM, and two kind of ANN namely the MLP and RBF structures were evolved to predict the relative humidity using wet bulb depression and dry bulb temperature. There are two parameters in the LSSVM which require being adjusted before the training step. The regularization parameter $(\gamma)$ and the kernel parameter corresponding to the kernel type $\left(\sigma^{2}\right)$ are the LSSVM tuned parameters. Moreover, the ANFIS method have some membership functions which have parameters. The optimal values of membership function parameters should be determined to reach the optimal ANFIS structure. The widely applied membership functions are the Generalized bell, Triangular, Gaussian, and Trapezoidal. In addition, the best number of hidden neurons in the structure of RBF and MLP artificial neural network should be used to obtain best values for weights and biases.

Consequently, the performance analysis should be performed to evaluate model's capability. In this regard, different statistical analyses such as the Average Absolute Error (AAE), Mean Squared Errors (MSEs), Mean Relative Errors (MREs), Root Mean Square Error (RMSE), Standard Deviations (STD), and the R-squared $\left(\mathrm{R}^{2}\right)$ were performed between the real and predicted data by the models. These analyses can be expressed as follows:

$$
\begin{aligned}
& M S E=\frac{1}{N} \sum_{i=1}^{N \sum_{1}}\left(\alpha c a l^{2}{ }_{\text {exp }}\right. \\
& M R E=\frac{1}{N} \sum_{i=1}^{N} \frac{\left|\alpha_{\exp }-\alpha_{c a l}\right|}{\alpha_{\exp }} \\
& A A E=\frac{1}{N} \sum_{i=1}^{N \sum}\left|\alpha c a l_{\text {exp }} \|\right| \\
& S T D_{\text {error }}=\left(\frac{1}{N-1} \sum_{i=1}^{N}(\text { error }-\overline{\text { error }})^{2}\right)^{0.5}
\end{aligned}
$$


$R M S E=\left(\frac{1}{N} \sum_{i=1}^{N \sum_{1}}\left(\alpha c a l^{2}{ }_{\exp }()^{0.5}\right)\right.$

Where $\alpha_{\exp }$ and $\alpha_{\text {cal }}$ stand for the experimental and determined data point by algorithms respectively. In addition, $\mathrm{N}$ stands for the number of data points.

\section{Results and Discussions}

We used the RBF as kernel function of the LSSVM method. The RBF parameters have a significant effect on the number of support vectors. The more the number of support vectors, the more the elapsed training time. The optimal values of the LSSVM parameters namely, the kernel parameter $\left(\sigma^{2}\right)$ and the regularization parameter $(\gamma)$ have been calculated using a genetic algorithm (GA). The GA is a straightforward strategy that uses concepts based on the natural selection for discovering the search space to reach a global optimum.

Moreover, the membership function implemented in the ANFIS algorithm has chosen the Gaussian function due to its high efficiency in practice. We implemented a combination of genetic algorithm and ANFIS to reach the best structure. Moreover, this work used 8 clusters for the ANFIS and the total number of membership function parameters obtained 48. Going to the clarification, each Gaussian membership functions have two parameters and the number of input and output variables are two (wet bulb depression and dry bulb temperature) and one (relative humidity) respectively. Thus, based on the 8 clusters and three variables, the overall number of membership function parameters is determined as follows:

Total Number of MF parameters $=($ Number of clusters $) \times($ Number of variables $) \times$ (number of MF parameter)

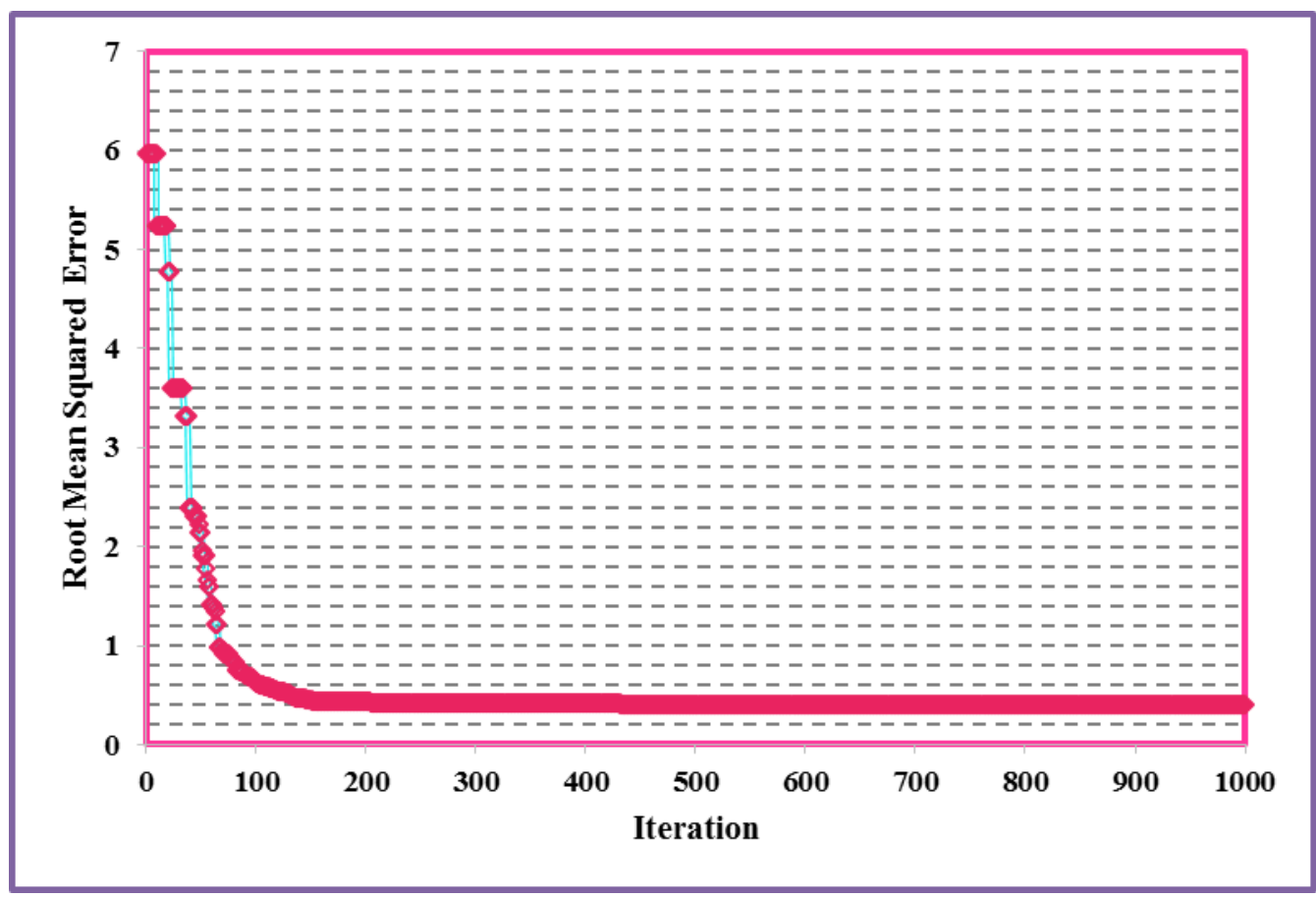


Fig. 5 RMSE for combination of GA and ANFIS during different iterations.

The performance of ANFIS based on RMSE of the determined and real values of humidity is shown in Fig. 5. As depicted, the maximum number of iterations was chosen 1000 and the optimum of RMSE was achieved as 0.40504. Fig. 6 illustrates trained membership function parameters for input variables including the wet bulb depression and dry bulb temperature, respectively. Their values vary between -1 and +1 , because of normalizing.

We used the linear transfer function and Log-Sigmoid transfer function in the output and hidden layers of suggested MLP-ANN model, respectively. In addition, we apply trial and error procedure to obtain an optimum number of neurons in its hidden layer. We used 7 neurons in the hidden layer and trained the MLP-ANN by the back-propagation technique. Table 2 presents obtained tuning parameters of weight and bias. Furthermore, as mentioned in theory section, the radial basis transfer functions (RBF) apply in the RBF-ANN model. The number of iterations is equal to the number of hidden neurons. Based on learning technique, the values of MSE was indicated in Fig. 7 for the duration of different iterations.

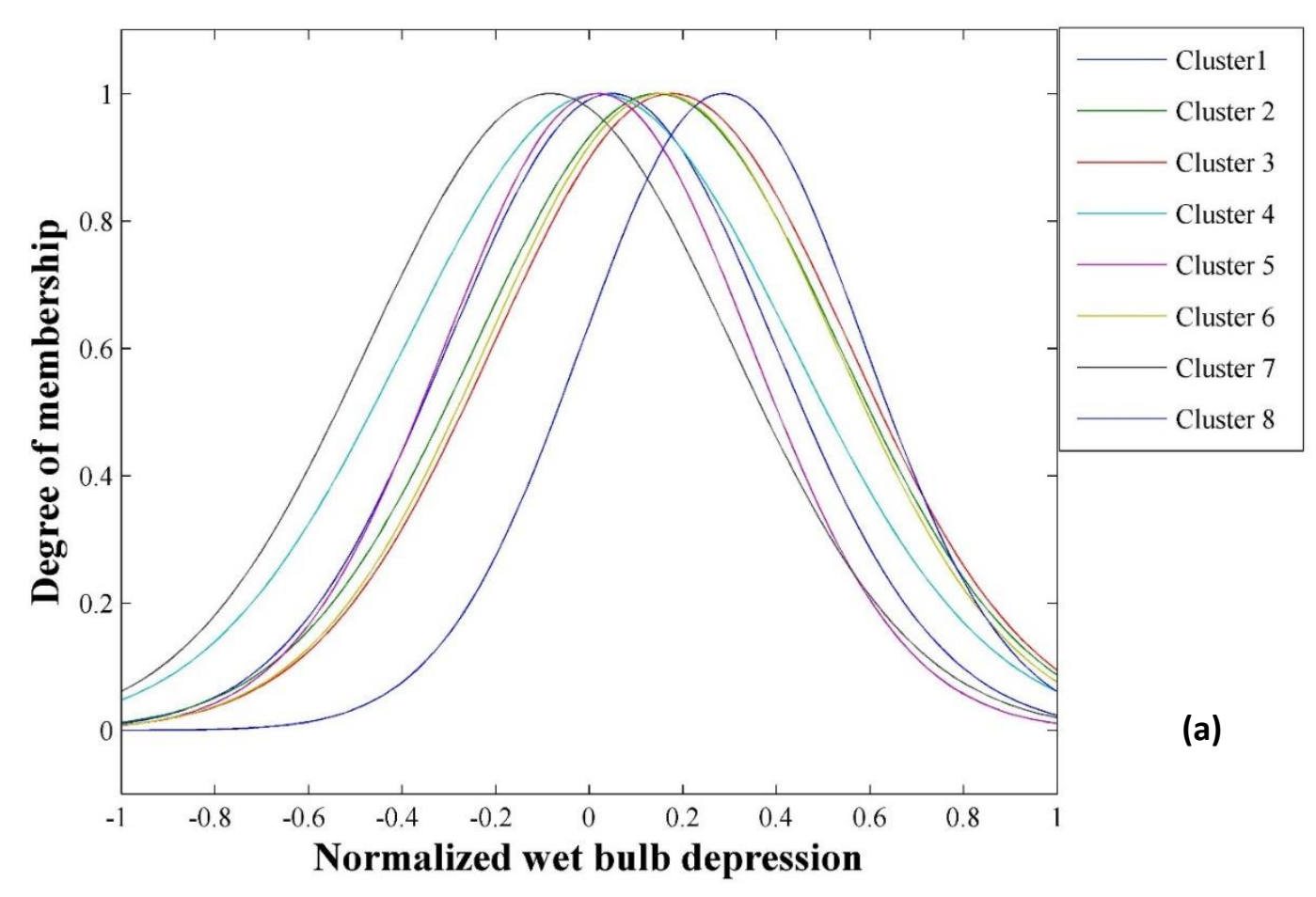




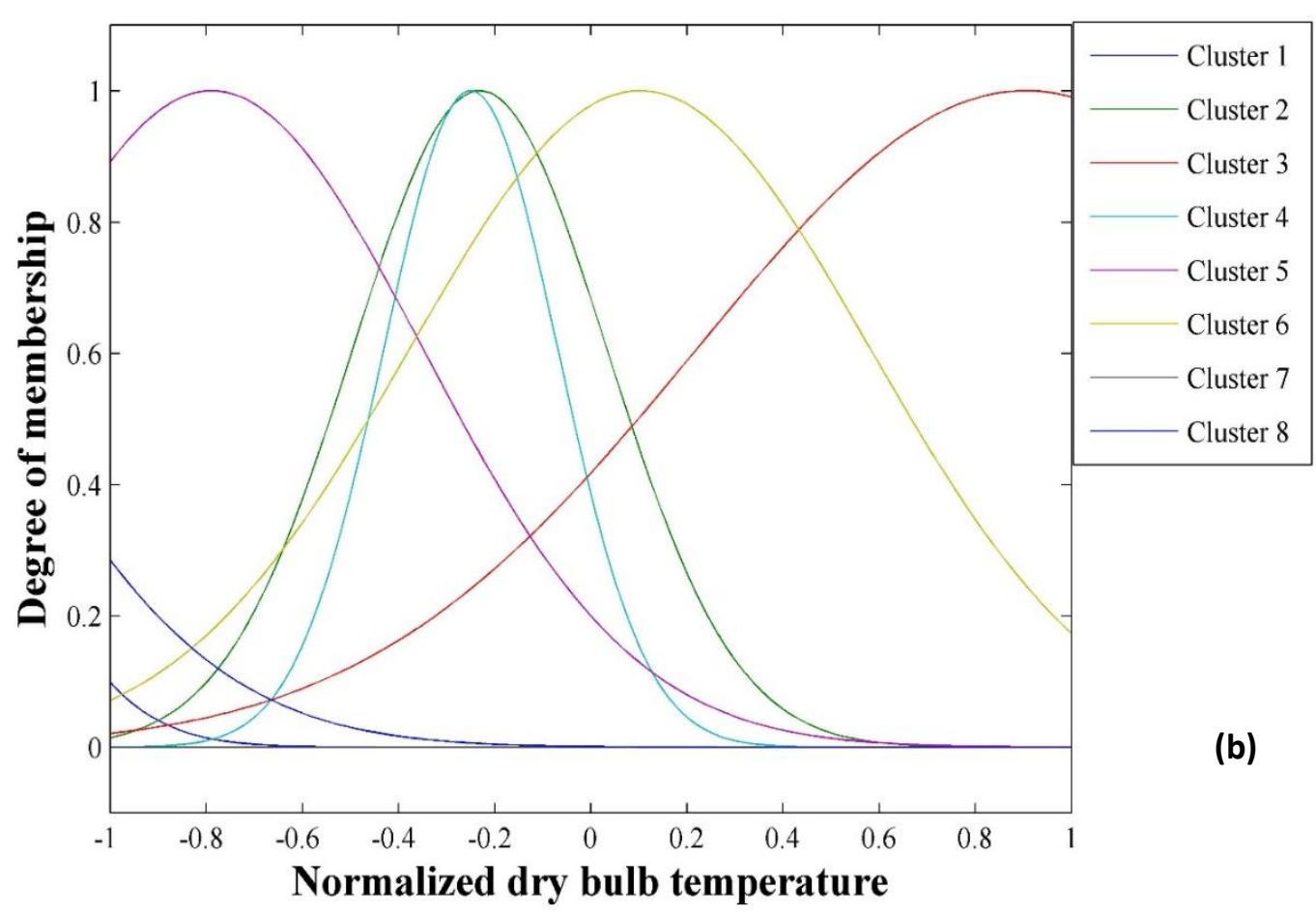

Fig. 6 The membership functions for (a) wet bulb depression and (b) dry bulb temperature.

Details of suggested algorithms have been reported in Table 3.

Table 2 Trained values of bias and weight for the MLP-ANN algorithm.

\begin{tabular}{cccccc}
\hline \multirow{2}{*}{ Neuron } & \multicolumn{3}{c}{ Hidden layer } & \multicolumn{2}{c}{ Output layer } \\
\cline { 2 - 5 } & \multicolumn{2}{c}{ Weight } & Bias & Weight & Bias \\
\cline { 2 - 5 } & $\begin{array}{c}\text { Wet bulb } \\
\text { depression }\end{array}$ & $\begin{array}{c}\text { Dry bulb } \\
\text { Temperature }\end{array}$ & $\mathbf{B}_{1}$ & Humidity & $\mathbf{B}_{2}$ \\
\cline { 2 - 5 } $\mathbf{1}$ & 348.1958 & 168.1785 & -75.9982 & 2.40597 & -548.86 \\
$\mathbf{2}$ & -692.671 & 539.7101 & 85.76041 & -0.76683 & \\
$\mathbf{3}$ & -75.0567 & -702.666 & 434.4219 & -0.16664 & \\
$\mathbf{4}$ & 0.31539 & -1.49156 & -3.57515 & 1879.579 & \\
$\mathbf{5}$ & -0.62346 & 2.066118 & 3.396896 & 550.1567 & \\
\hline
\end{tabular}




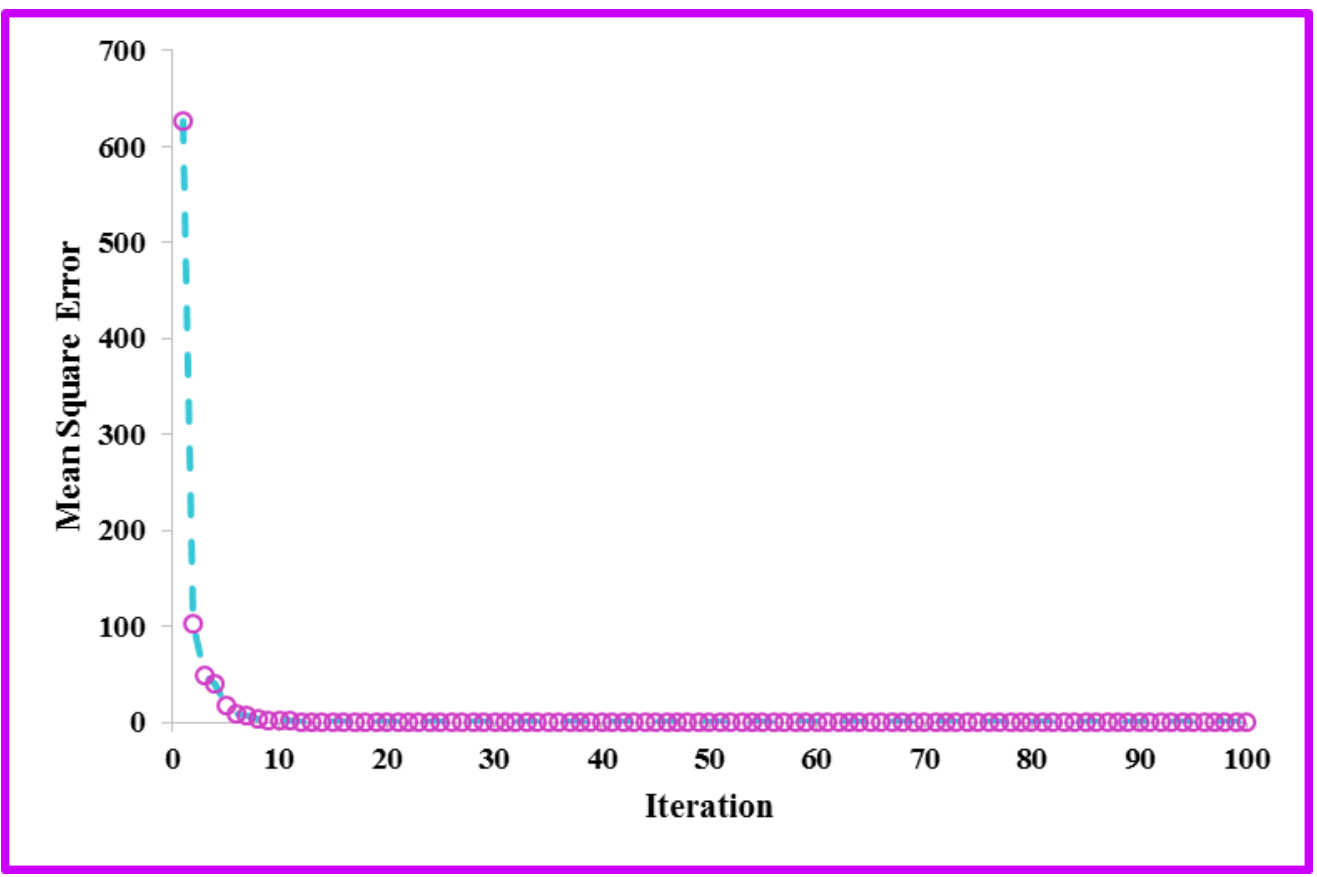

Fig. 7 Performance of RBF-ANN model at training stage.

Table 3 Details of the suggested models for the determination of relative humidity.

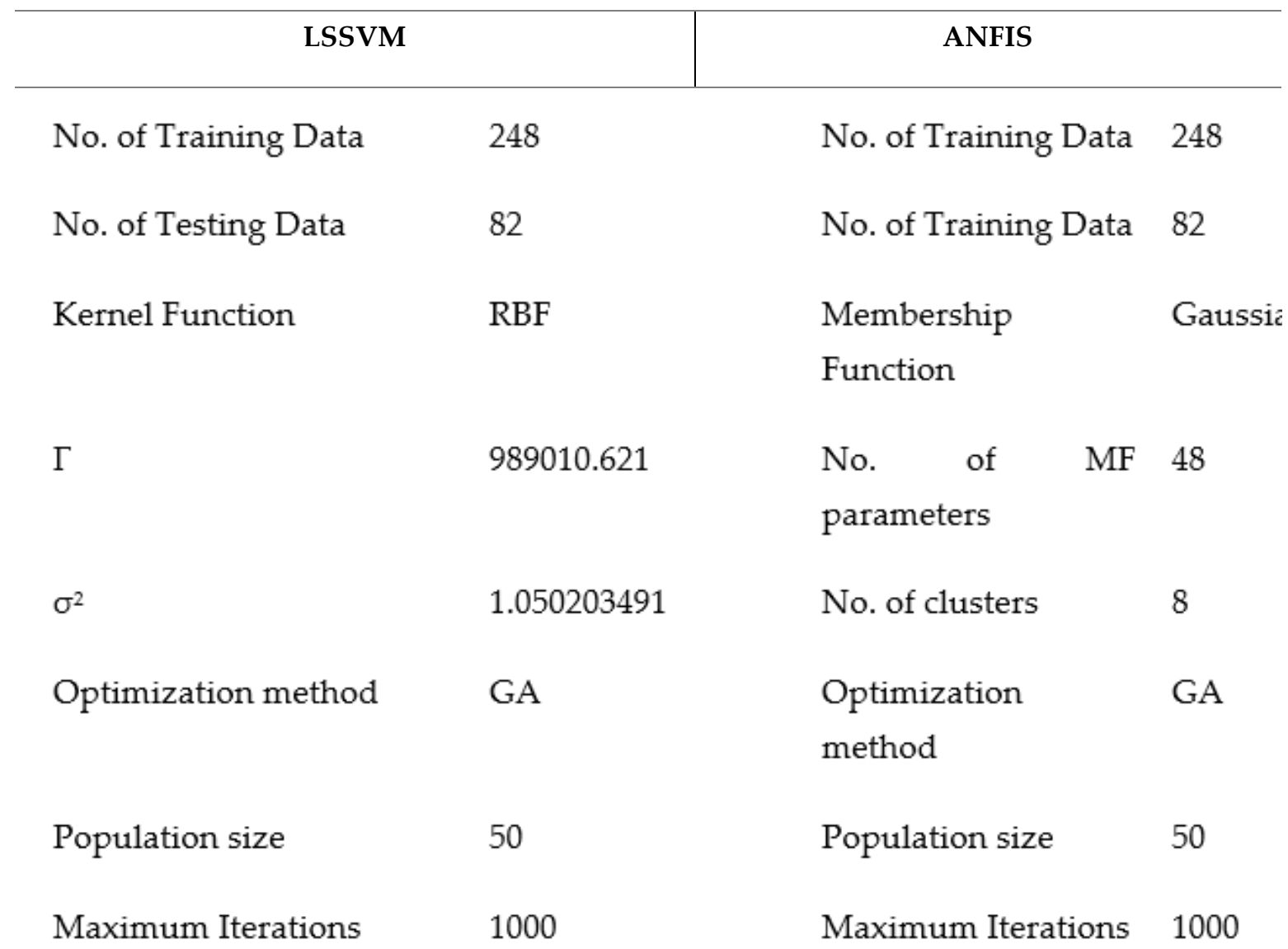




\begin{tabular}{|c|c|c|c|}
\hline \multicolumn{2}{|l|}{ MLP-ANN } & \multicolumn{2}{|c|}{ RBF-ANN } \\
\hline No. Input neuron layer & 2 & $\begin{array}{l}\text { No. Input } \\
\text { neuron layer }\end{array}$ & 2 \\
\hline No. Hidden neuron layer & 5 & $\begin{array}{l}\text { No. Hidden } \\
\text { neuron layer }\end{array}$ & 100 \\
\hline No. Output neuron layer & 1 & $\begin{array}{l}\text { No. Output } \\
\text { neuron layer }\end{array}$ & 1 \\
\hline Hidden layer activation function & Logsig & $\begin{array}{l}\text { Hidden layer } \\
\text { activation } \\
\text { function }\end{array}$ & RBF \\
\hline Output layer activation function & Purelin & $\begin{array}{l}\text { Output layer } \\
\text { activation } \\
\text { function }\end{array}$ & Purelin \\
\hline Number of data used for training & 248 & $\begin{array}{l}\text { Number of } \\
\text { data used for } \\
\text { training }\end{array}$ & 248 \\
\hline Number of data used for testing & 82 & $\begin{array}{l}\text { Number of } \\
\text { data used for } \\
\text { testing }\end{array}$ & 82 \\
\hline Number of max iterations & 1000 & $\begin{array}{l}\text { Number of } \\
\text { max } \\
\text { iterations }\end{array}$ & 403 \\
\hline
\end{tabular}

The actual against estimated relative humidity by the models have been illustrated in terms of wet bulb depression and dry bulb temperature in Fig. 8. In addition, the actual and predicted relative humidities are indicated simultaneously in Fig. 9 at training and testing stages. All models predict the relative humidity nicely and a reasonable agreement with the real data represents their capabilities. In Table 4, a comparison was done between the developed model by Bahadori et al. and our suggested models in this study. According to obtained outputs from the models, all approaches have a very good agreement with actual reported data and this comparison indicates their satisfactory performance. 
16 of 30

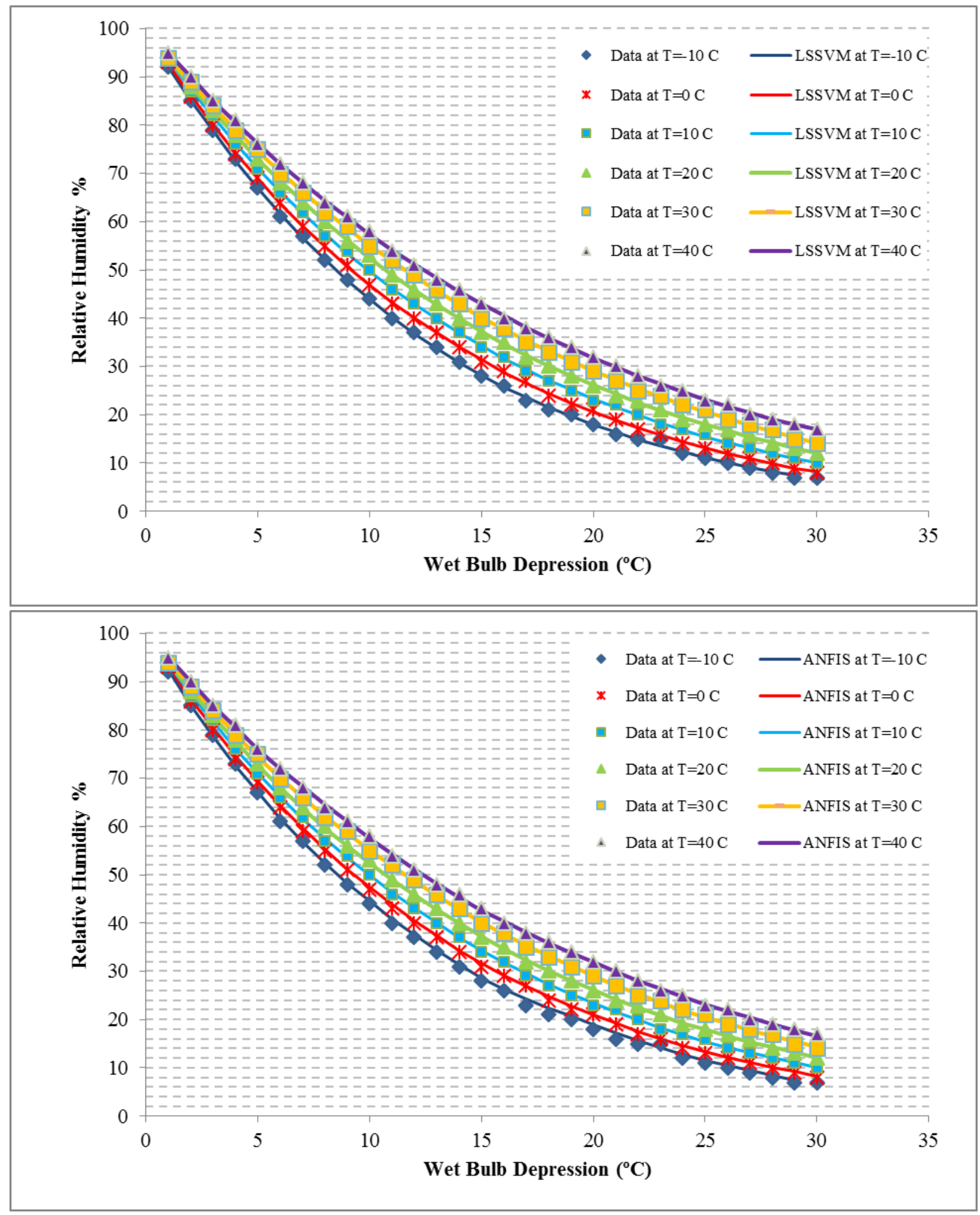


17 of 30
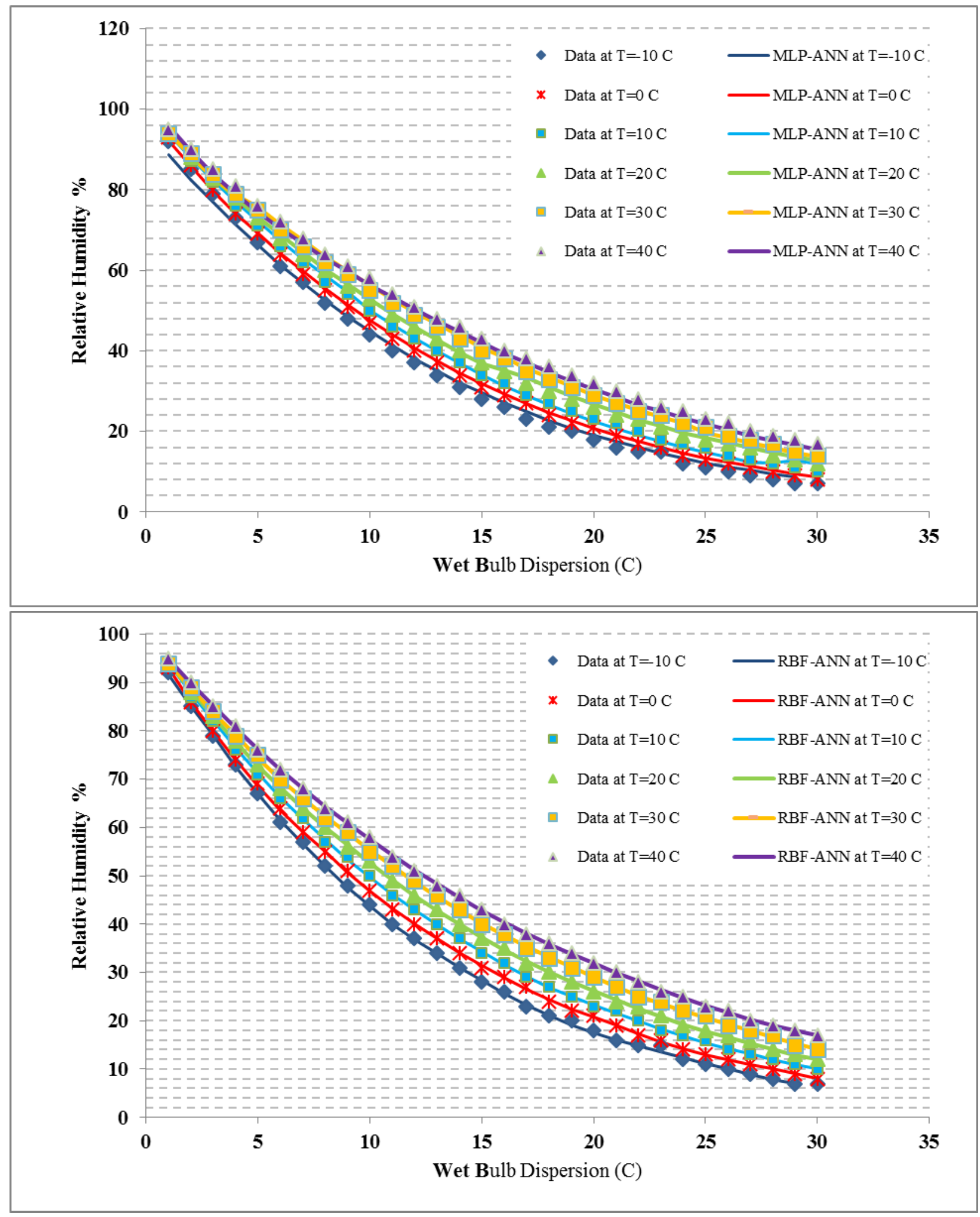

Fig. 8 The performance of predictive methods in comparison with the actual data for determining relative humidity. 

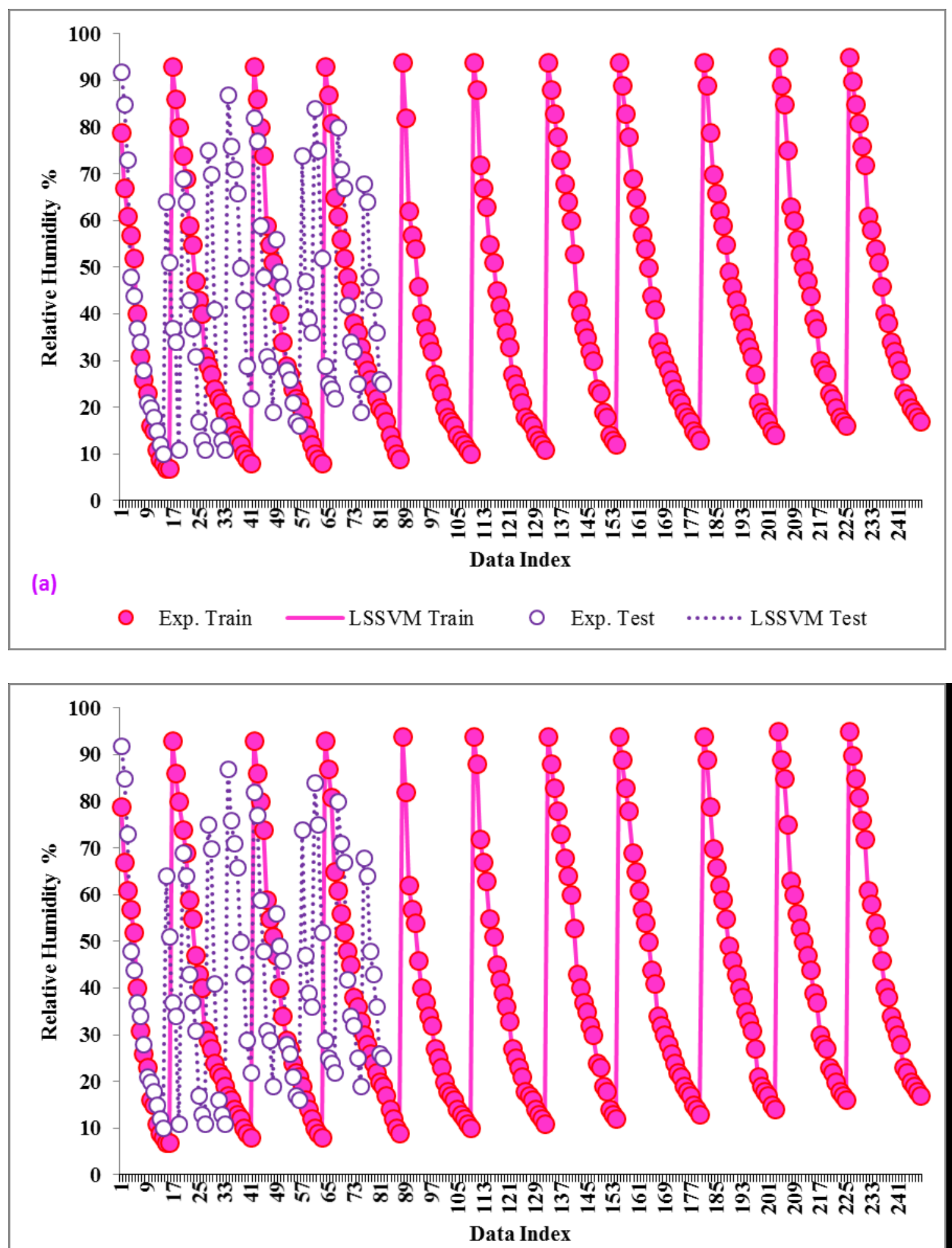

(b)

- Exp. Train $\longrightarrow$ ANFIS Train $O$ Exp. Test ANFIS Test 

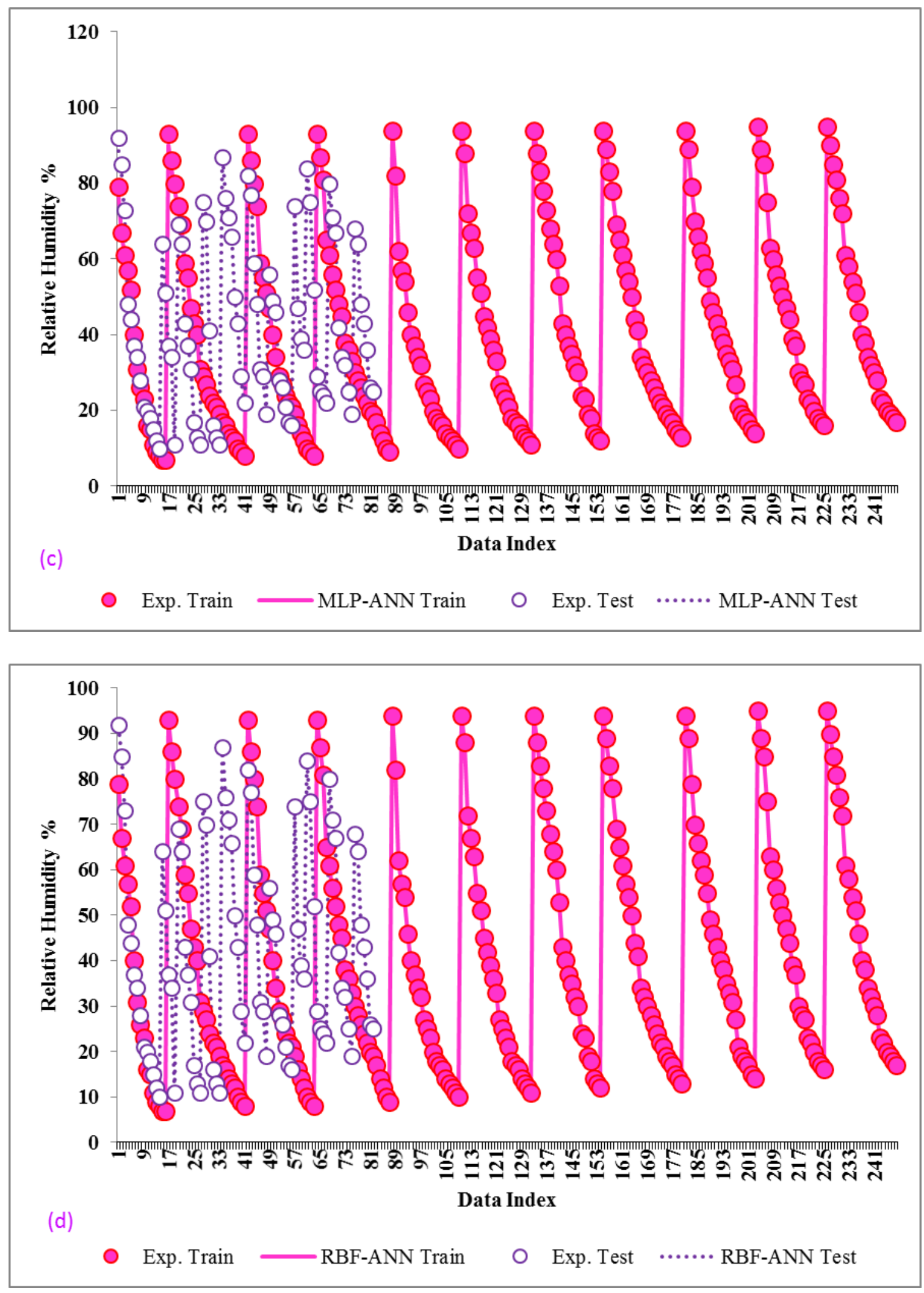

Fig. 9 Actual versus estimated relative humidity by: (a) LSSVM, (b) ANFIS, (c) MLP-ANN, and (d) RBF-ANN. 


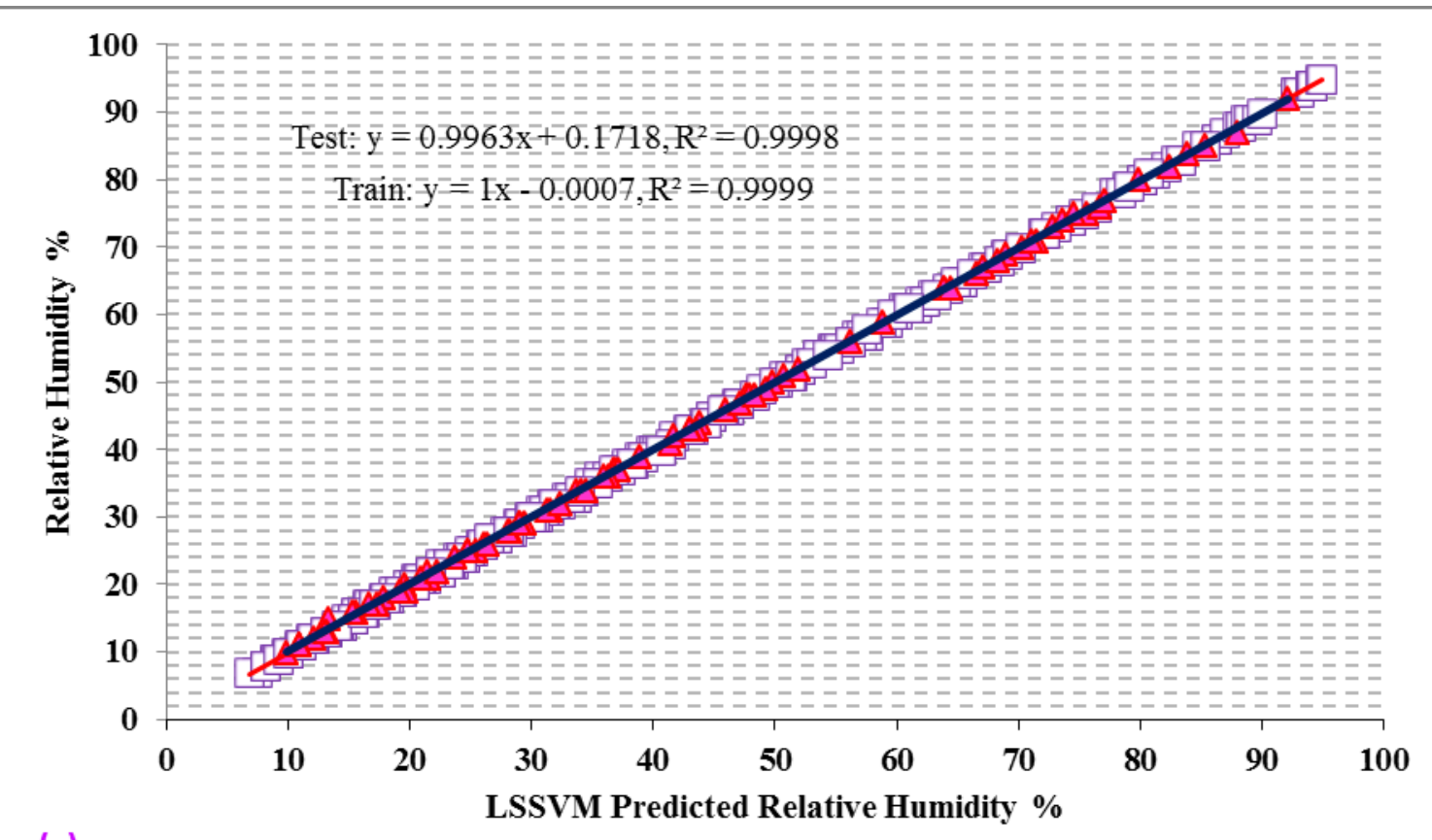

(a)
Training Data
$\Delta$ Testing Data Best Fit (Training Data) Best Fit (Testing Data)

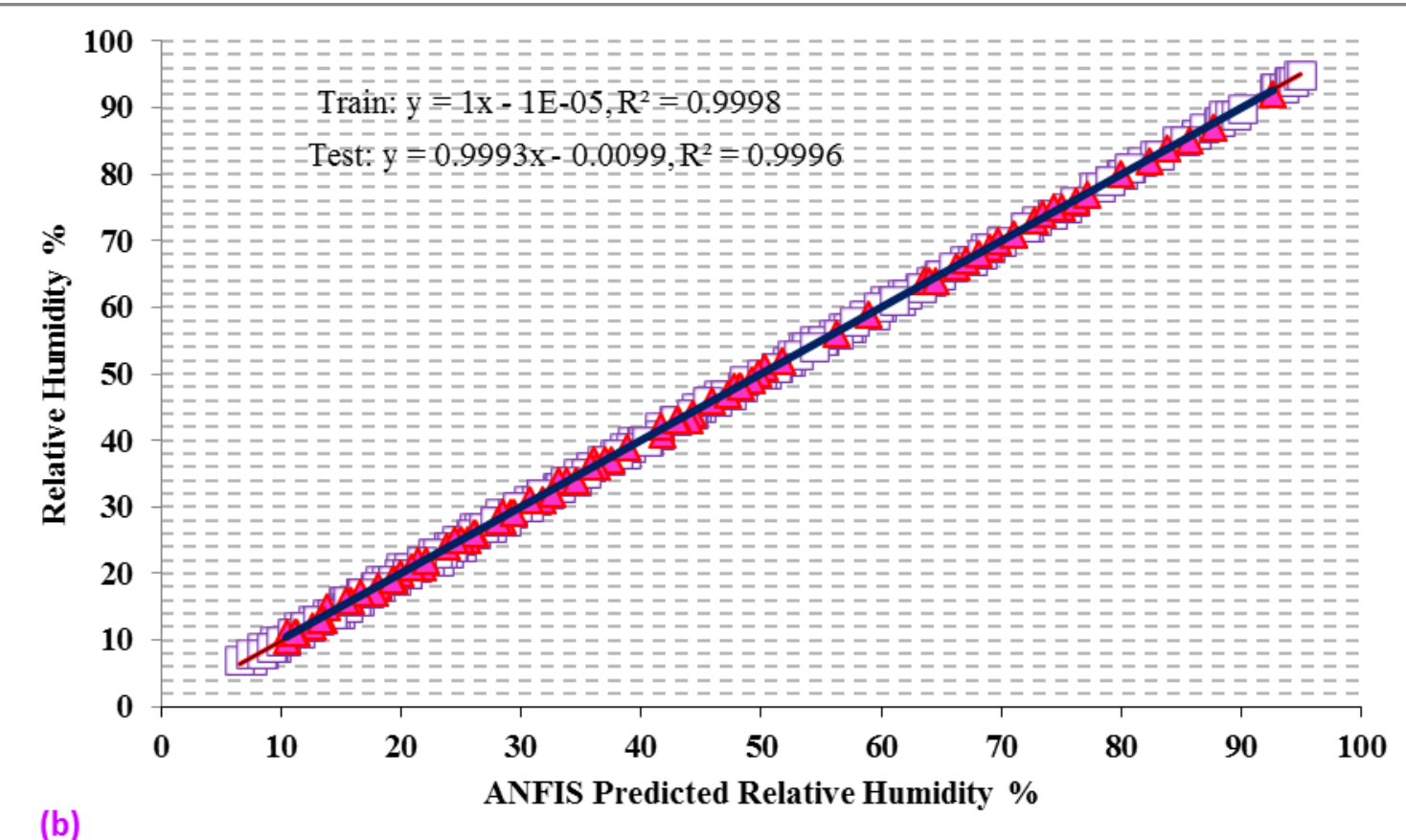

Training Data $\Delta$ Testing Data $\longrightarrow$ Best Fit (Training Data) $\longrightarrow$ Best Fit (Testing Data) 

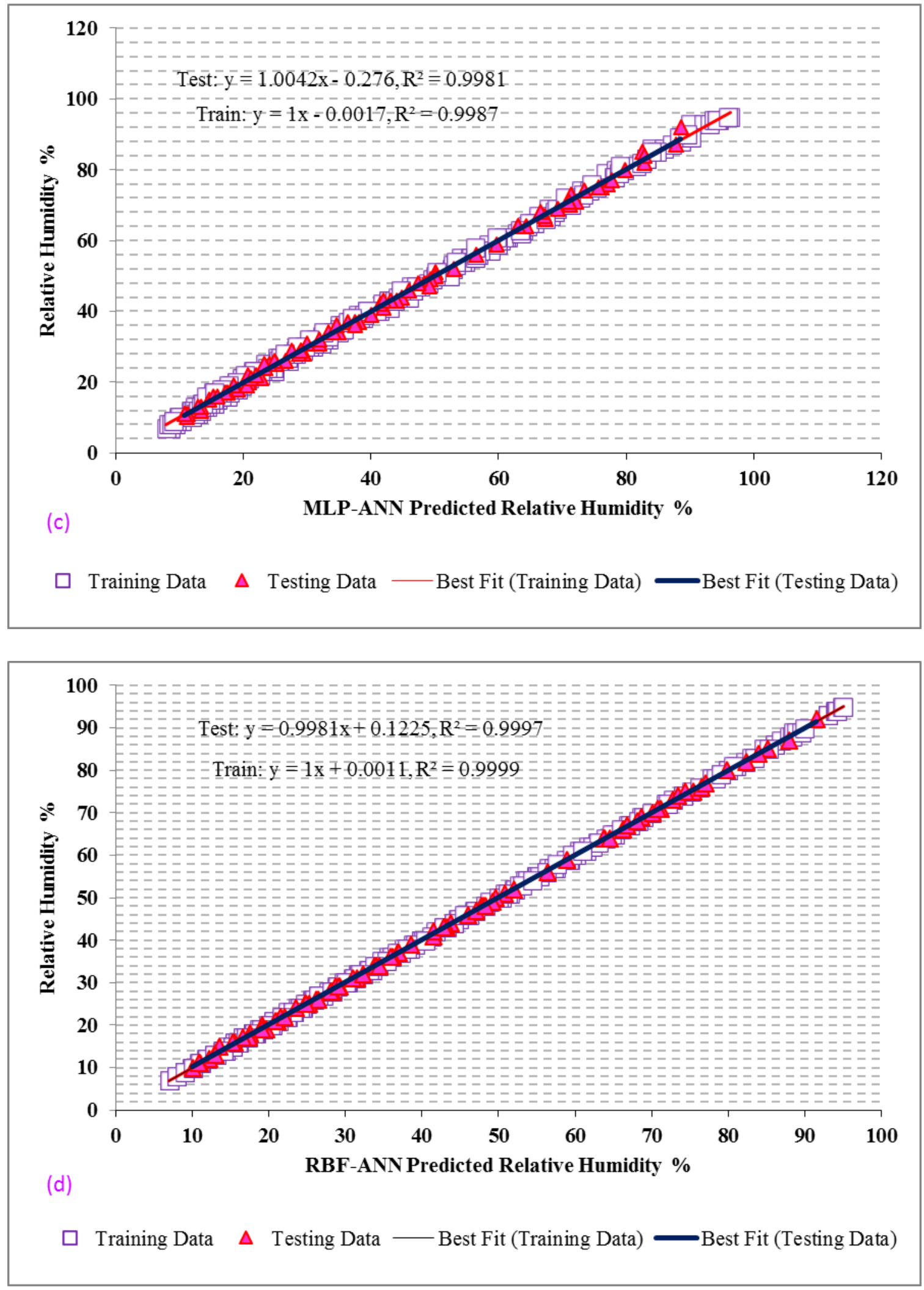

Fig. 10 Regression plots estimation of the relative humidity by: (a) LSSVM, (b) ANFIS, (c) MLPANN, and (d) RBF-ANN. 
The regression analysis has been shown in Fig. 10 for all models at training and testing phases. According to statistical knowledge, the $\mathrm{R}^{2}$ value is a well-known term which indicates the relationship between the model outputs and real values. Whereas $\mathrm{R}^{2}=1$, an interesting linear relationship is established between the predicted and real values. Conversely, as $\mathrm{R}^{2}$ is closer to zero, the linear relationship between the predicted and real values is weaker. A close-fitting of data points around the $45^{\circ}$ line for the predictive tools represent the precision.

Table 4 Comparison of the suggested algorithms with Bahadori et al. approach [9]

\begin{tabular}{|c|c|c|c|c|c|c|c|}
\hline $\begin{array}{c}\text { Temperature, } \\
\text { C }^{\mathrm{o}}\end{array}$ & $\begin{array}{c}\text { Wet bulb } \\
\text { depression, } \\
\text { C }^{-}\end{array}$ & $\begin{array}{c}\text { Reported } \\
\text { RH\% }\end{array}$ & $\begin{array}{c}\text { Bahadori } \\
\text { et al. } \\
\text { model }\end{array}$ & LSSVM & ANFIS & $\begin{array}{l}\text { RBF- } \\
\text { ANN }\end{array}$ & $\begin{array}{l}\text { MLP- } \\
\text { ANN }\end{array}$ \\
\hline-10 & 1 & 92 & 92.7 & 92.00 & 92.32 & 91.46 & 88.57 \\
\hline 0 & 2 & 86 & 86.4 & 86.18 & 86.44 & 86.06 & 85.57 \\
\hline 10 & 3 & 82 & 81.5 & 81.72 & 81.66 & 82.16 & 82.30 \\
\hline 20 & 4 & 78 & 77.6 & 77.82 & 77.65 & 77.76 & 78.06 \\
\hline 30 & 5 & 75 & 74.5 & 74.65 & 74.48 & 74.35 & 75.71 \\
\hline 40 & 6 & 72 & 72.1 & 72.04 & 72.05 & 72.04 & 70.43 \\
\hline-10 & 7 & 57 & 56.3 & 56.75 & 56.83 & 56.58 & 56.87 \\
\hline 0 & 8 & 55 & 54.6 & 54.80 & 55.25 & 54.94 & 55.54 \\
\hline 10 & 9 & 54 & 53.4 & 53.44 & 53.53 & 53.42 & 54.69 \\
\hline 20 & 10 & 53 & 52.4 & 52.50 & 52.42 & 52.71 & 52.76 \\
\hline 30 & 11 & 52 & 51.7 & 51.82 & 51.87 & 52.03 & 52.91 \\
\hline 40 & 12 & 51 & 51.1 & 51.17 & 51.21 & 51.24 & 50.29 \\
\hline-10 & 13 & 34 & 33.7 & 33.70 & 34.35 & 33.83 & 35.01 \\
\hline 0 & 14 & 34 & 33.9 & 34.00 & 34.38 & 34.15 & 34.61 \\
\hline 10 & 15 & 34 & 34.3 & 34.25 & 34.32 & 34.31 & 34.08 \\
\hline 20 & 16 & 35 & 34.8 & 34.72 & 34.69 & 34.67 & 35.04 \\
\hline 30 & 17 & 35 & 35.3 & 35.37 & 35.34 & 35.35 & 35.72 \\
\hline 40 & 18 & 36 & 35.7 & 35.92 & 35.89 & 35.89 & 34.68 \\
\hline-10 & 19 & 20 & 19.6 & 19.66 & 20.73 & 19.19 & 20.85 \\
\hline
\end{tabular}




\begin{tabular}{|c|c|c|c|c|c|c|c|}
\hline 0 & 20 & 21 & 20.5 & 20.56 & 21.02 & 19.02 & 19.06 \\
\hline 10 & 21 & 22 & 21.5 & 21.51 & 21.55 & 19.89 & 18.99 \\
\hline 20 & 22 & 23 & 22.6 & 22.56 & 22.48 & 20.82 & 21.26 \\
\hline 30 & 23 & 24 & 23.6 & 23.70 & 23.57 & 23.55 & 23.45 \\
\hline 40 & 24 & 25 & 24.6 & 24.70 & 24.74 & 24.81 & 23.29 \\
\hline-10 & 25 & 11 & 11 & 11.19 & 11.56 & 11.13 & 12.17 \\
\hline 0 & 26 & 12 & 11.9 & 11.92 & 12.22 & 11.84 & 12.39 \\
\hline 10 & 27 & 13 & 13 & 13.08 & 13.17 & 13.13 & 12.41 \\
\hline 20 & 28 & 14 & 14.2 & 14.25 & 14.30 & 14.07 & 14.82 \\
\hline 30 & 29 & 15 & 15.4 & 15.32 & 15.47 & 15.24 & 14.79 \\
\hline 40 & 30 & 17 & 16.8 & 16.89 & 16.54 & 17.01 & 15.49 \\
\hline
\end{tabular}

The obtained linear regressions of the LSSVM, ANFIS, MLP-ANN, and RBF-ANN from the statistical analyses were expressed respectively as follows:

$y=0.9963 x+0.1817 ; R^{2}=0.9998$

$y=0.9993 x-0.0099 ; R^{2}=0.9996$

$y=0.9963 x+0.1817 ; R^{2}=0.9998$

$y=0.9993 x-0.0099 ; R^{2}=0.9996$

Table 5 the statistical analyses of proposed models

\begin{tabular}{ccccccc}
\hline Model & & $\mathbf{R}^{2}$ & MSE & MRE (\%) & RMSE & STD \\
\hline LSSVM & Train & 0.9999 & 0.062767 & 0.731401 & 0.250534 & 0.250534 \\
& Test & 0.999757 & 0.131755 & 0.931285 & 0.36298 & 0.36266 \\
\multirow{2}{*}{ ANFIS } & Train & 0.999791 & 0.130927 & 1.198856 & 0.361839 & 0.361839 \\
& Test & 0.999627 & 0.193009 & 1.296151 & 0.439328 & 0.437627 \\
& Train & 0.998747 & 0.784485 & 2.730389 & 0.885711 & 0.885711 \\
& Test & 0.998085 & 1.001084 & 2.388525 & 1.000542 & 0.995979 \\
\hline
\end{tabular}




\begin{tabular}{lcccccc}
\hline RBF-ANN & Train & 0.999912 & 0.055023 & 0.644804 & 0.23457 & 0.234569 \\
& Test & 0.999746 & 0.134121 & 0.933355 & 0.366225 & 0.363983 \\
\hline
\end{tabular}

Besides above illustrations, the capability of aforementioned models was evaluated using the MSE, MRE, STD, RMSE, and $\mathrm{R}^{2}$. These statistical parameters have been summarized in Table 5 . Furthermore, the relative deviation percentages between the actual and estimated value of relative humidity by the models has been indicated in Fig. 11.
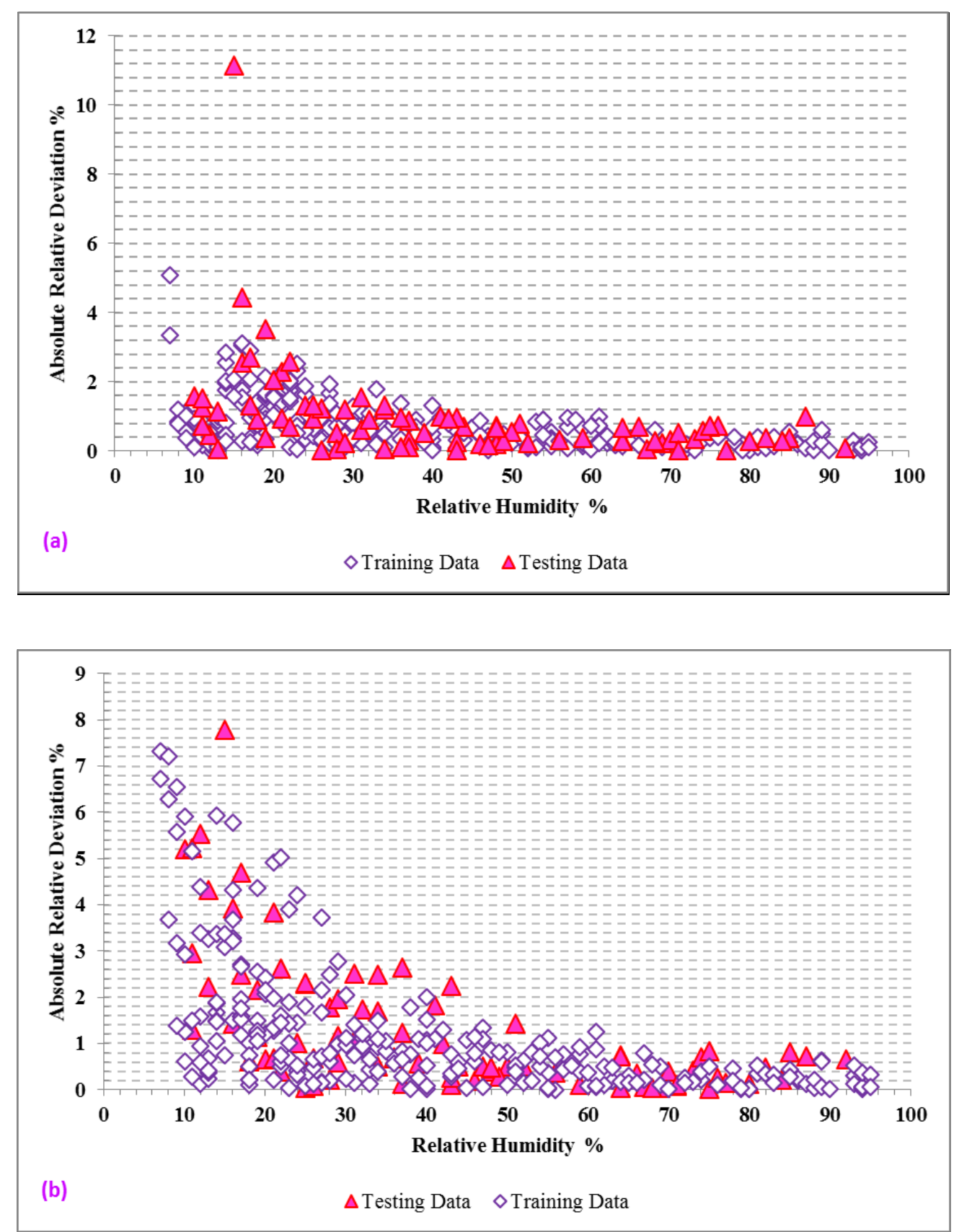


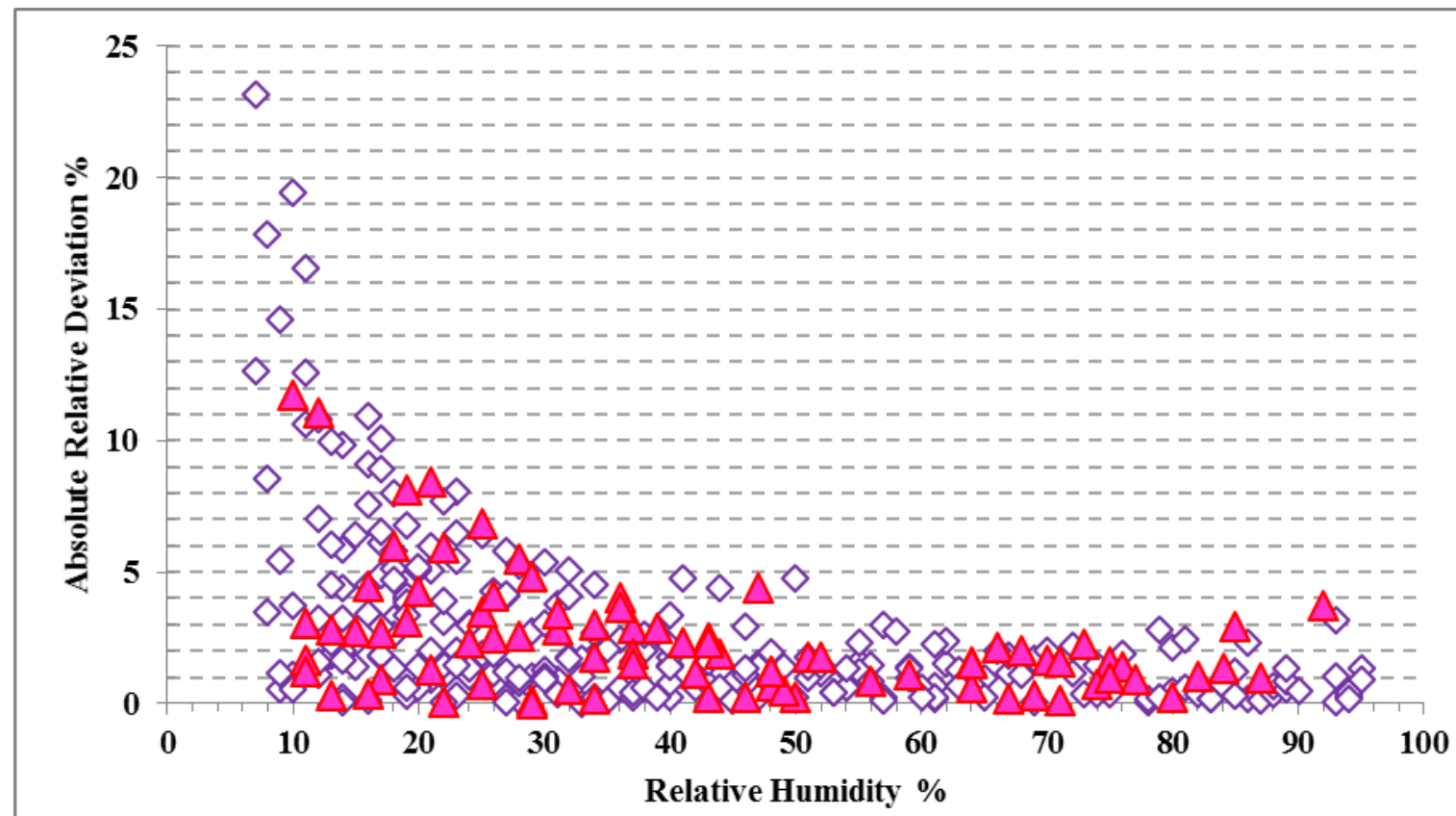

(c) $\quad \diamond$ Training Data $\Delta$ Testing Data

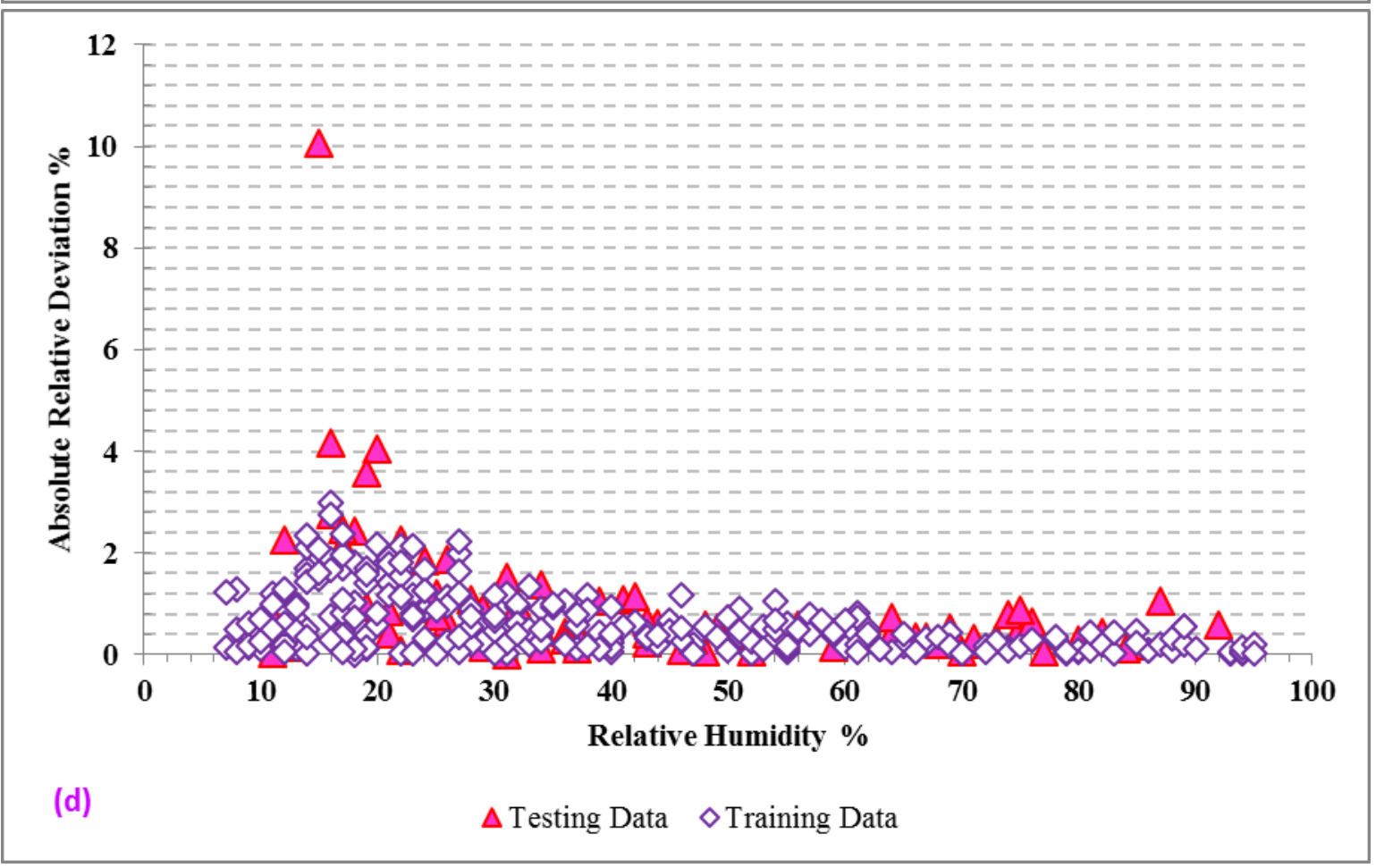

Fig. 11 The absolute relative deviation between the model outputs and actual values for: (a) LSSVM, (b) ANFIS, (c) MLP-ANN, and (d) RBF-ANN models. 

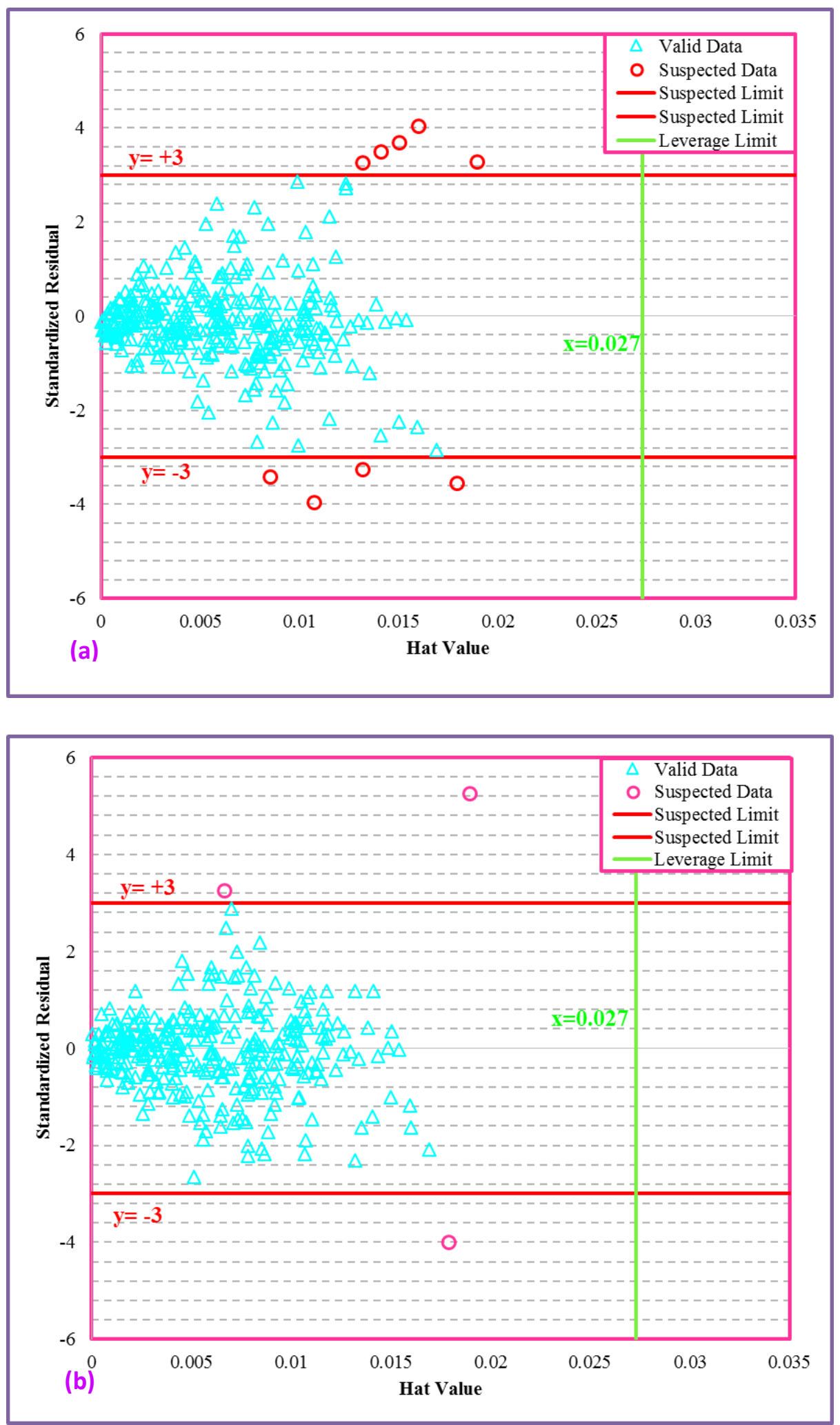

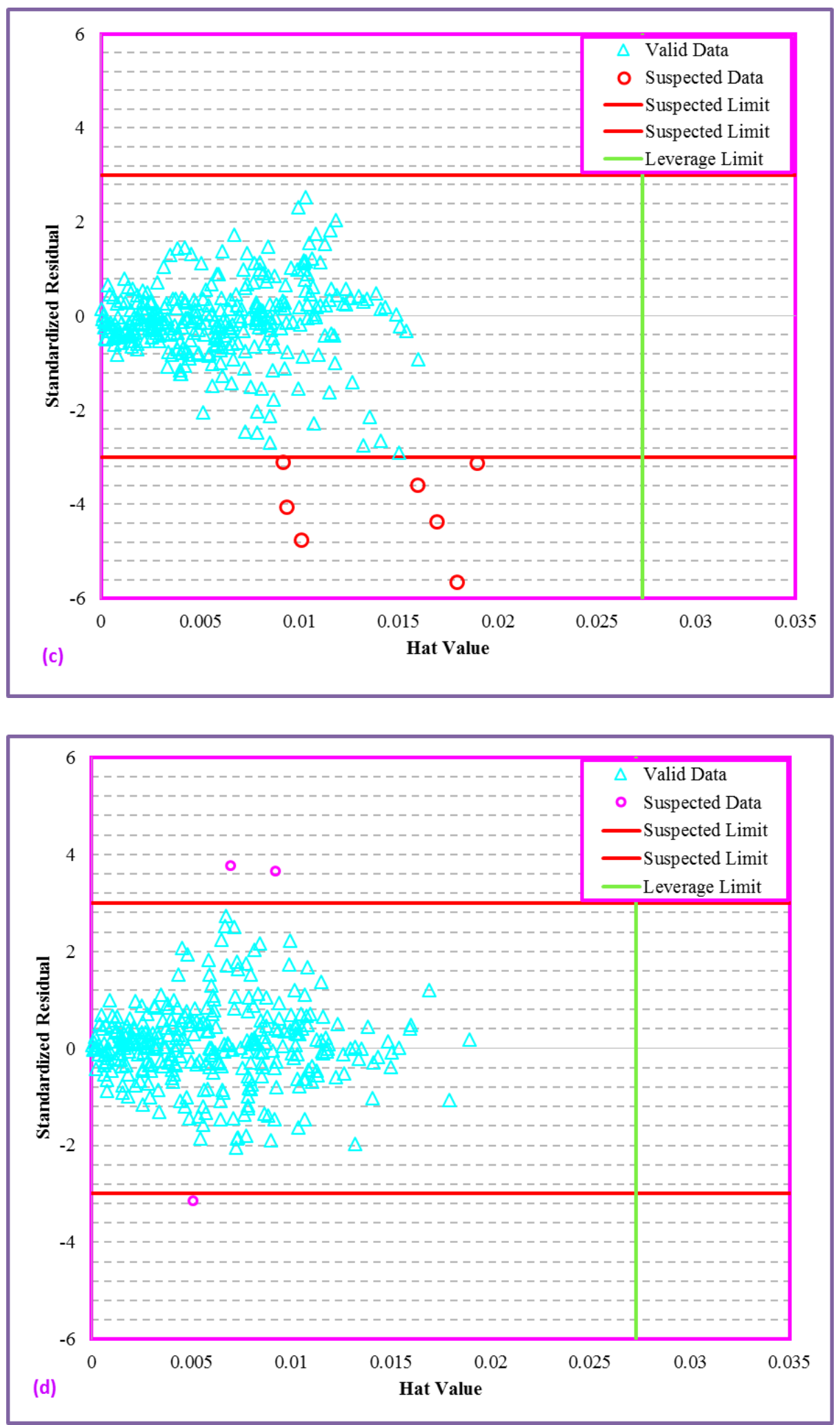

Fig. 12 William's plots for (a) LSSVM, (b) ANFIS, (c) MLP-ANN, and (d) RBF-ANN models.

In order to determine outliers of models, William's plot was utilized. This figure illustrates the standardized residuals versus hat values. Fig. 12 indicates the outlier analysis of the aforementioned models. Three limited boundaries can be obvious in these plots including leverage limit, upper and down suspected limit. Only the data with higher standardized residual values than 3 or lower than - 
3 are outliers and the data with hat $>$ hat ${ }^{*}$ are out of applicability domain of the applied models. The term hat* refers to warning leverage value and for the present models, the warning value obtained 0.027 . More information can be found in the literature regarding William's plot $[10,18,25]$.

Consequently, the ability of these proposed tools was significantly confirmed by the great agreement between the estimated data and the real data in evaluating the models for the training and testing stages.

\section{Conclusion}

The aim of this study was to investigate the potential of four models based on statistical learning concepts, such as ANFIS, LSSVM, RBF, and MLP artificial neural network for predicting relative humidity by dry bulb temperature and wet bulb depression. The membership function parameters of the ANFIS and the LSSVM parameters (i.e. the regularization parameter and kernel parameter) were determined through the genetic algorithm. The genetic algorithm had good performance to adjust their tuning parameters. Estimations were indicated to be in a close match with actual data points. Based on the results obtained by statistical analyses, the ability of the proposed methods was significantly confirmed by the good agreement between the estimated data and the real data in evaluating the models for the training and testing stages. In addition, outcomes of suggested models have been compared with another reported correlation and the accuracy of models was proved as expected. Unlike complex mathematical approaches for prediction of relative humidity, the suggested approaches are user-friend and would be of excellent help for scientists especially in cooling towers and air conditioning systems.

\section{References}

1. Przybylak, R. Air Humidity. In The Climate of the Arctic; Springer, 2016; pp. 127-136.

2. Vicente-Serrano, S.M.; Gimeno, L.; Nieto, R.O.; Azorin-Molina, C. Global Changes In Relative Humidity: Moisture Recycling, Transport Processes And Implications For Drought Severity. In Proceedings of the AGU Fall Meeting Abstracts; 2016.

3. Hasan, A. Indirect evaporative cooling of air to a sub-wet bulb temperature. Appl. Therm. Eng. 2010, 30, 2460-2468.

4. Shallcross, D.C. Preparation of psychrometric charts for water vapour in Martian atmosphere. Int. J. Heat Mass Transf. 2005, 48, 1785-1796.

5. Zhao, X.; Li, J.M.; Riffat, S.B. Numerical study of a novel counter-flow heat and mass exchanger for dew point evaporative cooling. Appl. Therm. Eng. 2008, 28, 1942-1951.

6. Mohanraj, M.; Jayaraj, S.; Muraleedharan, C. Applications of artificial neural networks for refrigeration, air-conditioning and heat pump systems-A review. Renew. Sustain. Energy Rev. 2012, 16, 1340-1358.

7. Hasan, A. Going below the wet-bulb temperature by indirect evaporative cooling: analysis using a modified $\varepsilon$-NTU method. Appl. Energy 2012, 89, 237-245. 
8. Singh, A.K.; Singh, H.; Singh, S.P.; Sawhney, R.L. Numerical calculation of psychrometric properties on a calculator. Build. Environ. 2002, 37, 415-419.

9. Bahadori, A.; Zahedi, G.; Zendehboudi, S.; Hooman, K. Simple predictive tool to estimate relative humidity using wet bulb depression and dry bulb temperature. Appl. Therm. Eng. 2013, $50,511-515$.

10. Baghban, A.; Bahadori, A.; Mohammadi, A.H.; Behbahaninia, A. Prediction of $\mathrm{CO}<\mathrm{inf}>2<$ inf $>$ loading capacities of aqueous solutions of absorbents using different computational schemes. Int. J. Greenh. Gas Control 2017, 57.

11. Baghban, A.; Bahadori, M.; Rozyn, J.; Lee, M.; Abbas, A.; Bahadori, A.; Rahimali, A. Estimation of air dew point temperature using computational intelligence schemes. Appl. Therm. Eng. 2016, 93.

12. Baghban, A.; Kardani, M.N.; Habibzadeh, S. Prediction viscosity of ionic liquids using a hybrid LSSVM and group contribution method. J. Mol. Liq. 2017, 236.

13. Baghban, A.; Mohammadi, A.H.; Taleghani, M.S. Rigorous modeling of $C O<\inf >2</$ inf $>$ equilibrium absorption in ionic liquids. Int. J. Greenh. Gas Control 2017, 58.

14. Baghban, A.; Pourfayaz, F.; Ahmadi, M.H.; Kasaeian, A.; Pourkiaei, S.M.; Lorenzini, G. Connectionist intelligent model estimates of convective heat transfer coefficient of nanofluids in circular cross-sectional channels. J. Therm. Anal. Calorim. 2017.

15. Burger, R.M. Engineering Design Handbook. Nat. Environ. Factors, Dep. Army, Richmond, VA 1975.

16. Jang, J.-S.R. Neuro-Fuzzy and Soft Computing/J.-SR Jang, C.-T. Sun, E. Mizutani. A Comput. Approach to Learn. Mach. Intell. Saddle River, NJ Prentice Hall, Inc., 1997.-614 p 1997.

17. Jang, J.-S.R.; Sun, C.-T.; Mizutani, E. Neuro-fuzzy and soft computing; a computational approach to learning and machine intelligence. 1997.

18. Baghban, A.; Sasanipour, J.; Haratipour, P.; Alizad, M.; Vafaee Ayouri, M. ANFIS modeling of rhamnolipid breakthrough curves on activated carbon. Chem. Eng. Res. Des. 2017, 126.

19. Buragohain, M.; Mahanta, C. A novel approach for ANFIS modelling based on full factorial design. Appl. Soft Comput. 2008, 8, 609-625.

20. Suykens, J.A.K.; Van Gestel, T.; De Brabanter, J. Least squares support vector machines; World Scientific, 2002; ISBN 9812381511.

21. Ye, J.; Xiong, T. Svm versus least squares svm. In Proceedings of the International Conference on Artificial Intelligence and Statistics; 2007; pp. 644-651.

22. Mitchell, T.M. Artificial neural networks. Mach. Learn. 1997, 45, 81-127.

23. Dayhoff, J.E.; DeLeo, J.M. Artificial neural networks. Cancer 2001, 91, 1615-1635. 
24. Park, J.; Sandberg, I.W. Approximation and radial-basis-function networks. Neural Comput. 1993, 5, 305-316.

25. Baghban, A.; Mohammadi, A.H.; Taleghani, M.S. Rigorous modeling of CO 2 equilibrium absorption in ionic liquids. Int. J. Greenh. Gas Control 2017, 58, $19-41$. 